\title{
Identification of SPRR3 as a novel diagnostic/prognostic biomarker for oral squamous cell carcinoma via RNA Sequencing and Bioinformatic analyses
}

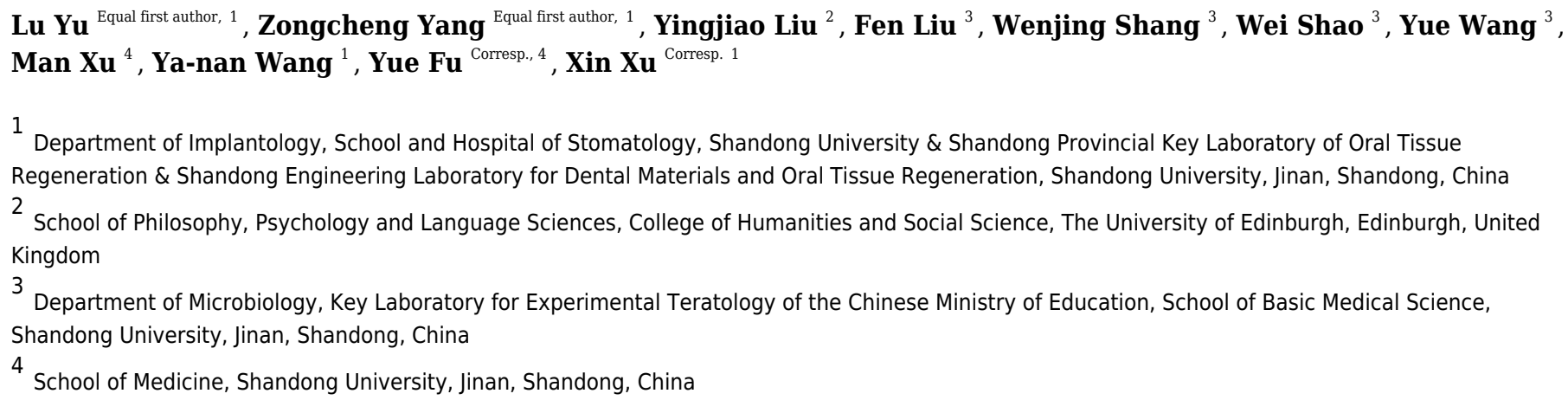

Oral squamous cell carcinoma (OSCC) has always been one of the most aggressive and invasive cancers among oral and maxillofacial malignancies. As the morbidity and mortality of the disease have increased year by year, the search for a promising diagnostic and prognostic biomarker for the disease is becoming increasingly urgent. Tumorous and adjacent tissues were collected from three OSCC sufferers, and we obtained 229 differentially expressed genes (DEGs) between tumor and normal tissues via highthroughput RNA sequence. Function and pathway enrichment analyses for DEGs were conducted to find a correlation between tumorigenesis status and DEGs. Protein interaction network and molecular complex detection (MCODE) were constructed to detect core modules. Two modules were enriched in MCODE. The diagnostic and prognostic values of the candidate genes were analyzed, which provided evidence for the candidate genes as new tumor markers. SPRR3, a potential tumor marker that may be useful for the diagnosis of OSCC, was screened out. The survival analysis showed that SPRR3 under expression predicted the poor prognosis of OSCC patients. Further experiments have also shown that the expression of SPRR3 decreased as the malignancy of OSCC increased. Therefore, we believe that SPRR3 could be used as a novel diagnostic and prognostic tumor marker. 


\section{Identification of SPRR3 as a novel diagnostic/prognostic biomarker}

\section{2 for oral squamous cell carcinoma via RNA Sequencing and}

\section{Bioinformatic analyses}

4 Lu Yu${ }^{1}$, Zongcheng Yang ${ }^{1}$, Yingjiao Liu ${ }^{2}$, Fen Liu ${ }^{3}$, Wenjing Shang ${ }^{3}$, Wei Shao ${ }^{3}$, Yue Wang

$5 \quad$ Man $\mathrm{Xu}^{4}$, Yanan Wang ${ }^{1}$, Yue $\mathrm{Fu}^{4}$, Xin $\mathrm{Xu}^{1}$

$6{ }^{1}$ Department of Implantology, School and Hospital of Stomatology, Shandong University \&

7 Shandong Provincial Key Laboratory of Oral Tissue Regeneration \& Shandong Engineering

8 Laboratory for Dental Materials and Oral Tissue Regeneration, No.44-1 Wenhua Road West, Jinan

9 250012, Shandong, PR China

$10{ }^{2}$ School of Philosophy, Psychology and Language Sciences, College of Humanities and Social 11 Science, the University of Edinburgh, Edinburgh EH8 9AD, United Kingdom

$12{ }^{3}$ Department of Microbiology, Key Laboratory for Experimental Teratology of the Chinese 13 Ministry of Education, School of Basic Medical Science, Shandong University, Jinan, Shandong 14 250012, PR China

$15{ }^{4}$ School of Medicine, Shandong University, Jinan, Shandong 250012, PR China

17 Correspondence:

19 Yue $\mathrm{Fu}$

20 fuyuesdu@163.com

$21 \mathrm{Xin} \mathrm{Xu}$

22 xinxu@sdu.edu.cn

LY and ZY contributed equally to this article. 


\section{Abstract}

Oral squamous cell carcinoma (OSCC) has always been one of the most aggressive and invasive cancers among oral and maxillofacial malignancies. As the morbidity and mortality of the disease have increased year by year, the search for a promising diagnostic and prognostic biomarker for the disease is becoming increasingly urgent.

Tumorous and adjacent tissues were collected from three OSCC sufferers, and we obtained 229 differentially expressed genes (DEGs) between tumor and normal tissues via high-throughput RNA sequence. Function and pathway enrichment analyses for DEGs were conducted to find a correlation between tumorigenesis status and DEGs. Protein interaction network and molecular complex detection (MCODE) were constructed to detect core modules. Two modules were enriched in MCODE. The diagnostic and prognostic values of the candidate genes were analyzed, which provided evidence for the candidate genes as new tumor markers.

SPRR3, a potential tumor marker that may be useful for the diagnosis of OSCC, was screened out. The survival analysis showed that SPRR3 under expression predicted the poor prognosis of OSCC patients. Further experiments have also shown that the expression of SPRR3 decreased as the malignancy of OSCC increased. Therefore, we believe that SPRR3 could be used as a novel diagnostic and prognostic tumor marker.

\section{Introduction}

Oral squamous cell carcinoma (OSCC) is a well-known malignancy that frequently arises from the epidermal layer in oral cavity and, in most of the cases, develops from precancerous lesions of the oral mucosa. The majority of the OSCC patients are among those affected by head and neck squamous cell carcinoma (HNSCC), with an overall 5-year survival rate of $\sim 64.4 \%$. The outcome of OSCC treatment may vary depending on a series of factors, such as age, race, tumor staging, secondary complications, and location of the tumor in the oral cavity (Zanoni et al., 2019). So far, current therapeutic strategies have been unable to fundamentally predict and fully cure OSCC patients. The mechanisms involved in the transformation of the normal oral epithelium into OSCC have not been fully characterized (Abdalla et al., 2017), therefore limiting any prediction on tumor progression and potential therapeutic approaches. Despite the various methods used for identifying the tumor type/stage and corresponding prognosis, most of these approaches have limited use to 
60 precisely specify the occurrence and development of the disease. Due to postoperative recurrence

61 and lack of targeted chemical drugs, further innovations are urgently needed in regard to OSCC 62 diagnosis and treatment. Therefore, a better prediction method for OSCC prognosis has been 63 proposed, namely using gene expression data combined with clinical information of affected 64 patients. Since microarray analyses and high-throughput sequencing techniques have been largely utilized in oncology, a representative number of genetic and molecular alterations have been reported (Shridhar et al., 2016). Still, the use of single markers to accurately diagnose malignant tumors is still challenging. Thus, it appears critical to collectively distinguish candidate genes that

In general terms, Bioinformatics refers to a cutting-edge method of biological information analysis with obvious advantages when compared with traditional trials, such as the high efficiency to analyze extensive amounts of supporting data. As a result, Bioinformatic approaches have been continuously applied in cancer research to eliminate inconsistent results led by small sample size and/or by applying different technological platforms. However, the application of Bioinformatic techniques in the field of OSCC research is still restricted, despite the multitude of available information awaiting to be mined in public databases. Instead of performing isolated bioinformatics analysis, we have presently added biopsy RNA sequencing from OSCC patients during the analytical process. We further confronted this analysis with large sampling of microarray data in the current databases, accompanied with immunohistochemical staining, therefore increasing the reliability of endpoint results. According to this Bioinformatic workflow, SPRR3 (Small Proline Rich Protein 3) was screened out as a potential prognostic and diagnostic biomarker for OSCC.

SPRR3 is a protein coding gene largely expressed in normal epithelial cells, whose gene product is known as a small proline-rich protein typically present in epidermal differentiation complexes

84 (EDCs). Importantly, SPRR3 has been recognized as a marker of terminal squamous cell differentiation (Candi, Shmidt, \& Melino, 2005), but its role in OSCC has been rarely studied. In our current work, we have established a bioinformatic-based workflow (Fig. 1) to screen novel diagnostic and prognostic OSCC markers as well as to verify their predictive efficiency in OSCC patients. As a result, we were able to identify SPRR3 as a putative target marker in OSCC.

\subsection{Information of specimens}


93 We collected three pairs of tumor and adjacent oral normal specimens from three patients 94 diagnosed with OSCC, whose diagnoses were supported by pathological examination results. All 95 the three patients, coming from Stomatological Hospital of Shandong University, were considered 96 as candidates for curative surgical resection (patients' details in Table S1). This study was 97 approved by the Research Ethics Committee of Stomatological Hospital of Shandong University 98 (No.20190205). Tissue microarray chips consisted of 61 OSCC samples and 10 samples of normal control was constructed by Shanghai Qutdo Biotech Company (Shanghai, China. Patients' details in Table S2). The tissue microarray construction was approved by Taizhou Hospital Research Ethics Committee in Zhejiang Province (YB M-05-01). All experiments were performed complying with the relevant regulations, and written informed consent was obtained from patients.

\subsection{High-throughput RNA sequence (RNA-seq)}

105

106

107

108

109

110

111

112

113

114

115

116

117

118

119

120

121

122

123

124

125

126

127

We performed poly-A sequencing on the three pairs of tissues. According to the manufacturer's protocol, we extracted total RNA from the three pairs of tissues we collected mentioned above by Trizol (invitrogen), and removed ribosomal RNA by the Ribo-Zero ${ }^{\mathrm{TM}}$ kit (Epicentre, Madison, WI, USA). The first and second strand cDNA synthesis of the fragment RNA (the average length of $200 \mathrm{bp)}$ was performed, followed by adaptor ligation, and low cycle enrichment in accordance with the instructions of NEBNext ${ }^{\circledR}$ Ultra ${ }^{\mathrm{TM}}$ RNA Library Prep Kit for Illumina (New England Biolabs, Ipswich, MA, USA). In addition, the clean data quality control was filtered using Trimmomatic (Version 3). We utilized the Agilent 2200 TapeStation (Life Technologies, Waltham, MA, USA) and Qubit ${ }^{\circledR} 2.0$ (Invitrogen, Carlsbad, California, USA) to evaluate the purified library products. The libraries were paired-end sequenced (PE150, Sequencing reads were $150 \mathrm{bp}$ ) at Guangzhou RiboBio Co., Ltd. (Guangzhou, Guangdong province, China) using IlluminaHiSeq 3000 platform (NEB).

Paired-end reads alignment to the human reference genome hg19 (NCBI build 37.1, http://www.ncbi,nlm.nih.gov/projects/genome/assembly/grc/) were performed with HISAT2 (http://ccb.jhu.edu/software/hisat2/manual.shtml\#getting-started-with-hisat2, Pertea, Kim, Pertea, Leek, \& Salzberg, 2016). And we use HTSeq v0.6.0 (Anders, Pyl, \& Huber, 2015) to count the reads numbers mapped to each gene. The expression levels were presented as RPKM (expected number of Reads PerKilobase of transcript sequence per Million base pairs sequenced), which was the recommended and most frequently-used measuring unit. And the RNA-seq data was uploaded to GEO (Gene Expression Omnibus) database. (GSE140707, https://www.ncbi.nlm.nih.gov/geo/query/acc.cgi?acc=GSE140707) 


\subsection{Data processing for public databsets}

129

130 GEPIA (Gene Expression Profiling Interactive Analysis) (Tang et al., 2017) is an interactive web server for analyzing the RNA-seq expression data from the TCGA (the Cancer Genome Atlas) (https ://tcga-data.nci.nih.gov/tcga/) (Tomczak, Czerwinska, \& Wiznerowicz, 2015) and

133 Genotype-Tissue Expression (GTEx) projects. GEPIA provides customizable functions such as tumor/normal differential expression analysis, profiling according to cancer types or pathological stages, patient survival analysis, etc.

GSE3524 (Toruner et al., 2004), GSE42743 (Lohavanichbutr et al., 2013) and GSE30784 (Chen et al., 2008) datasets in the GEO database were selected as data source for OSCC information. Among them, GSE30784 and GSE3524 are the datasets containing gene expression information in both tumor and normal oral tissues, and GSE42743 is the one containing patients' survival and clinical data such as survival time, live status, and other related factors that may influence the development and occurrence of OSCC. All of the datasets were selected to increase the sample size of the RNA-seq microarray data, so as to better verify the differential expression and diagnose the prognosis effect of the candidate genes. The platform files and original files (.CEL files) were downloaded. With the aid of Robust Muliti-array Average (RMA) algorithm, data imputation, background correction, $\log _{2}$ conversion, quantile normalization, pro summarization and missing value supplementation were performed on the matrix data of each GEO dataset through the "impute" package and "affy" package (Gautier, Cope, Bolstad, \& Irizarry, 2004) in R/Bioconductor software (Szklarczyk et al., 2011) (version 3.5.3). A collection of 306 specimens of OSCC was obtained from TCGA database, the level 3 RNA sequence data (.count files) was downloaded from TCGA website (https://portal.gdc.cancer.gov/), and their correlated clinical information from FireBrowse database (http://www.firebrowse.org/) (Chabanais, Labrousse, Chaunavel, Germot, \& Maftah, 2018). The RNA sequence data in the TCGA database was normalized and processed using the "edgR" package (Zhang et al., 2019) prior to $\log _{2}$ conversion (Z. Yang et al., 2019).

\subsection{Differential expression analysis}

All the DEGs were obtained through high-throughput RNA-seq. The statistically significant DEGs were acquired by utilizing the "DEseq" package (Wang, Feng, Wang, Wang, \& Zhang, 2010), and set adjusted P-value (FDR) $<0.05$ and $\mid \log 2$ (fold change (FC)) $\mid>1$ as inclusion criteria. Then a hierarchical clustering analysis was performed using the "gplots" package (Ma, Wang, Liu, Xia, 
$162 \&$ Tao, 2017) in R/Bioconductor software based on the expression level of DEGs in different 163 groups, and colors represent different clustering information.

\subsection{Construction of a protein-protein interaction (PPI) network and module analysis}

166

167 To assess the inter-relationships among DEGs, at protein level, these were mapped using the 168 Search Tool for the Retrieval of Interacting Genes (STRING) database (Szklarczyk et al., 2017).

169 The inter-relation among DEGs (combined score $\geq 0.4$ ) was utilized to design a PPI network using 170 Cytoscape (Shannon et al., 2003) (v3.6.1). The molecular complex detection (MCODE) plugin 171 was used to select meaningful modules in the PPI network. For this, MCODE k-core $>2$ and 172 MCODE score $>5$ were utilized as cut-off values. MCODE is a widely used algorithm for 173 predicting molecular complexes, according to the inter-connectivity and density between nodes in 174 PPI networks. The respective score determines the number of included nodes in clusters as well as 175 the cluster size. An appropriate score is more likely to help select the meaningful cluster, which 176 bears potential biological functions. The k-core is a vertex weighting method. The weighting 177 scheme further boosts the weight of densely connected vertices, to further optimize the module178 based cluster scoring system. A higher k-core of a particular network is the central and most 179 densely connected subnet (Bader \& Hogue, 2003).

\subsection{Kyoto Encyclopedia of Genes and Genomes (KEGG) pathway and Gene Ontology (GO) enrichment analysis}

To determine the functions of respective DEGs, we initially performed a GO enrichment analysis (Liu, Liu, \& Rajapakse, 2018) via the Database for Annotation, Visualization and Integrated Discovery (DAVID) database (Version 6.8, https://david.ncifcrf.gov/) (Huang da, Sherman, \& Lempicki, 2009). The enrichment of different pathways was mapped using the KEGG pathway analysis (Kanehisa et al., 2017). An FDR < 0.05 was set as an inclusion criterion in both GO and KEGG enrichment analyses. In order to identify putative roles of distinct gene modules in OSCC, we performed the GO enrichment analysis for DEGs that composed each selected module cluster. The "cluster Profiler" package in R/Bioconductor software (Yu, Wang, Han, \& He, 2012) was utilized for this enrichment analysis. The FDR $<0.05$ was set again as an inclusion criterion in this

193 GO analysis. 
195

196

197

198

199

200

201

202

203

204

205

206

207

208

209

210

211

212

213

214

215

216

217

218

219

220

221

222

223

224

225

226

\subsection{Immunohistochemical (IHC) staining}

The included patients' clinical and pathological data were shown in Table S3. The tissue microarray chips were dewaxed and dehydrated, and then incubated overnight at $4{ }^{\circ} \mathrm{C}$ with monoclonal rabbit anti-human SPRR3 (Abcam, ab218131) after epitope retrieval, $\mathrm{H}_{2} \mathrm{O}_{2}$ treatment and non-specific antigens blocking, after signals detection by using DAB staining kit (Vector Laboratories, Burlingame, CA, USA), we incubated chips with the secondary antibody. IHC analysis of the chips were performed under optical microscopes of $100 \times$ and $400 \times$.

\subsection{Gene-set enrichment analysis (GSEA)}

GSEA is a computer-based tool used for microarray data analysis and annotation, which is based on biological knowledge (Aravind Subramanian, 2005). The GSEA enrichment score (ES) reflects the degree to which a particular set is over-represented at the extremes (top or bottom) of the entire ranking list. ES is normalized for each geneset to account for the overall size of the set, yielding a normalized enrichment score (NES). In this study, the data of OSCC was respectively obtained from our own RNA-seq microarray data and TCGA database. RNA-seq samples were grouped as tumoral and normal tissues. Samples from TCGA database were grouped according to the quantile of SPRR3 expression levels. Thereafter, samples were sorted from low to high in terms of expression level, where the first quantile was defined as the "low expression group" while the last quantile was defined as the "high expression group". GSEA annotated the GO, KEGG, and Hallmark enrichment results of these genes with an enrichment score. The inclusion criteria of the enriched results were established as $|\mathrm{NES}|>1$, P-value $<0.05$, and FDR $<0.25$ (Subramanian et al., 2005).

\subsection{Statistical analysis}

To explore the connection between factors and overall survival (OS) of the OSCC patients, univariate Cox regression model was utilized in specimens of GSE42743. Subsequently, we used significant factors to conduct the Least Absolute Shrinkage and Selection Operator (LASSO) method. LASSO regression contributes to variable selection and regularization, at the same time that it fits the generalized linear model. Using this statistical approach, we could also avoid over- 
227 fitting at some extent. Because the complexity of LASSO regression was controlled by the 228 coefficient $(\lambda)$, a less variable model was obtained by performing a penalty ratio, according to their 229 size. A relatively small number of highly correlated indicators was then obtained and, 230 subsequently, a plot was designed according to the partial possibility deviance vs $\log (\lambda)$. The 231 positive factors of the LASSO regression analysis were incorporated into the multivariant Cox 232 regression model, which confirmed the feasibility of these factors as independent predictors of 233 OSCC. We later verified the effect(s) in the TCGA database using this same methodology.

234 The Kaplan Meier (K-M) survival analysis was performed to examine the relationship between the survival time of OSCC patients and the expression level of respective DEGs. OSCC samples from

236

237

238

239

240

241

242

243

244

245

246

247

248

249

250

251

252

253

254

255

256

257

258

259

GSE42743 and TCGA were divided into 2 groups, according to the quartile of expression for each selected gene. As previously, samples were sorted from low to high in terms of expression level for each target gene. First and last quantiles were related to the low and high expression groups, respectively. We calculated and then indicated the hazard ratio (HR) with $95 \%$ confidence intervals and log-rank P-value along the plot.

To evaluate the discriminatory accuracy of a selected gene between two groups, its expression level was included in the Receiver Operating Characteristic (ROC) curve analysis. The ROC curve is an essential tool for diagnostic detection or biomarker prediction in a certain disease (Matthews, Ranson, \& Kelso, 2011). Plots of sensitivity (true-positive fraction) versus 1-specificity (falsepositive fraction) were constructed. The value for the area under ROC curve (AUC) corresponds to the ability of one gene to distinguish tumor tissues from adjacent ones. $\chi^{2}$ test was utilized to verify the correlation between gene expression levels and clinicopathologic factors. In this context, samples were also grouped according to the quantile of expression level of a respective target gene. All statistical analyses were conducted by SPSS 25.0 software (SPSS Inc., Chicago, IL, USA). The correlation coefficient between two genes was calculated by Pearson correlation analysis. For expression data comparison, student's t test (two-tailed) and ANOVA were performed using GraphPad Prism 8 software.

\section{$3 \quad$ Results}

\subsection{DEGs search and analysis}

According to the sequencing data here available, a total of 229 DEGs were identified in OSCC samples, from which 85 genes were up-regulated while 144 were down-regulated. The most common up- $\left(\log _{2} \mathrm{FC}>1\right.$, FDR $\left.<0.05\right)$ and down-regulated $\left(\log _{2} \mathrm{FC}<-1\right.$, FDR $\left.<0.05\right)$ genes are 
260 listed in Table S4. Both volcano plot and heatmap were generated to show the discrepancy among

261 the expressed genes when comparing OSCC and adjacent normal tissues (Figs. 2A-B).

\section{$263 \quad 3.2$ The enrichment results of the DEGs}

265 Based on the GO enrichment analysis, the commonly enriched categories were (i) keratinization, 266 (ii) keratinocyte differentiation, (iii) cell adhesion, (iv) migration and (v) proliferation. In addition, 267 DEGs were enriched in alcohol and drug metabolic processes (Fig. 2C), which have been closely 268 related to both tumorigenesis and development. The most stringent results of GO enrichment 269 analysis, including BP (biological process), MF (molecular function) and CC (cellular component) terms, (the top 100 items of each terms with the lowest FDR) are presented in Table S5.

KEGG pathway analysis showed that the majority of DEGs were enriched in (i) drug metabolism, (ii) retinol metabolism, (iii) chemical carcinogenesis and (iv) leukocyte transendothelial migration pathways (Fig. 2D). Detailed results of KEGG enrichment analysis are presented in Table S6.

In order to validate the DEG-related functions and pathways on an unbiased basis, GSEA analysis

275

276

277

278

279

280

281

282

283

284

285

286

287

288

289

290 was performed using previously generated RNA-seq data. DEGs were mostly enriched in (i) epithelial mesenchymal transition (EMT), (ii) fatty acid metabolism, (iii) K-Ras signaling down and (iv) TGF- $\beta$ signaling. In regard to the GO-BP terms, GSEA showed enrichment of DEGs in (i) DNA replication, (ii) epidermal cell differentiation and (iii) positive regulation of cell activation. In the context of KEGG enrichment, DEGs were largely present in (i) drug metabolic cytochrome P450, (ii) extracellular matrix receptor interaction, (iii) focal adhesion and (iv) tight junction (Figs. 2E-G). Above all, DEGs were more concentrated in biological processes including (i) epidermal cell differentiation, (ii) metabolic process and (iii) cell adhesion, reiterating a potential correlation with tumorigenesis and cancer development.

\subsection{Identification of module clusters via PPI network}

A global PPI network was designed after establishing the inter-relationships among the annotated DEGs (STRING database search). As a result, 166 nodes with a combined score $>0.4$ were obtained (Fig. S1). MCODE plugin was applied for module analysis, where two modules were chosen for further analysis according to the inter-connectivity and density between nodes in PPI 
291 networks. Module clusters 1 and 2 consisted, respectively, of eight and ten nodes (Figs. 3A-B).

292 All DEGs present in these two particular modules are shown in Table 1.

293 Genes from selected module clusters were included in the GO enrichment analysis to properly 294 understand their functions and gene product attributes. In regard to BP, the DEGs from module 295 cluster 1 were correlated with (i) keratinocyte differentiation, (ii) epidermal cell differentiation and 296 (iii) epidermis development. In terms of CC, the DEGs were enriched in cornified envelope and 297 desmosomes, and for MF, the DEGs were enriched in protein binding, bridging and molecular 298 adaptor activity (Fig. 3C-E). The DEGs in module cluster 2 were mainly correlated with collagen 299 metabolic process and extracellular matrix organization (Fig. 3F-H). Interestingly, most of the 300 genes here involved (for instance, MMP (Matrix Metallopeptidase) family members) have been 301 associated with tumor invasion and metastasis (Lu et al., 2017; Zou et al., 2019). The MCODE 302 score of module cluster 1 was higher, and the hub genes in this cluster were barely reported in 303 OSCC studies. Hence, the module cluster 1 was preliminarily chosen for subsequent evaluation.

\subsection{Validation of DEGs in independent OSCC datasets}

According to the TCGA database, OSCC accounts for the majority of HNSCC cases. The RNA expression level of DEGs in module cluster 1 was preliminarily analyzed by GEPIA in the HNSCC dataset. Among these DEGs, CSTA (Cystatin A), EVPL (Envoplakin), PPL (Periplakin), SPRR3, TGM1 (Transglutaminase 1) were significantly down-regulated in tumor tissues when compared with their normal counterparts $(\mathrm{P}<0.05)$ (Figs. 4A-H). To further demonstrate that these five genes are differentially expressed in OSCC, we utilized the GSE30784 and GSE3524 datasets to confirm potential disease-related DEGs. Consistently, these five genes were significantly down regulated in tumor tissues (Figs. 4I-M for GSE30784; Figs. S2A-F for GSE3524).

\subsection{Identification of DEG-related prognostic value}

318 To properly select the predictive factors associated with the overall survival of OSCC patients, the 319 five above-mentioned genes and clinical disease characteristics were introduced into univariate 320 Cox regression model using the GSE42743 dataset. The most statistically significant DEGs 321 (SPRR3, PPL, TGM1) and N staging were concomitantly examined by LASSO method. As a 322 result, only SPRR3 and N staging were confidently sorted out (Figs. 5A-B). To further determine 323 whether low SPRR3 expression could be an independent predictor of OSCC prognosis, a 
324 multivariate Cox regression model was executed. As predicted, multivariate Cox regression 325 analysis showed that SPRR3 expression levels ( $\mathrm{HR}=0.865,95 \% \mathrm{CI}$ : 0.754-0.992, P-value $=$ 326 0.037) act as an independent prognostic factor for the OS of OSCC patients (Table 2).

327 The SPRR protein family has been reported to be functional in a variety of tumors (Specht et al., 328 2013). Still, the expression and function of SPRR 3 in OSCC remain largely unclear. Thus, we have 329 presently proposed SPRR3 as an OSCC-related gene and attempted to explore its role in 330 tumorigenesis and development of this condition. Therefore, K-M survival analysis was carried 331 out using the GSE42743 dataset to verify whether SPRR3 could act as a prognostic marker (log rank P-value $<0.05$; Fig. 5C).

333 Statistical platforms including univariate Cox regression model, LASSO method and Cox multivariate regression model were also approached to validate the SPRR3's prognostic value, based on the TCGA database. According to the univariate Cox analysis, factors including low SPRR3 expression, complete tumor remission, lymphovascular and perineural invasion, as well as $\mathrm{N}$ staging were significantly associated with a poor prognosis. SPRR3 was also screened out by the LASSO method (Figs. 5D-E). Multivariate Cox proportional regression analysis revealed that SPRR3 expression levels $(\mathrm{HR}=0.544,95 \% \mathrm{CI}$ : 0.329-0.899, P-value $=0.017)$ was an independent prognostic factor for the OS of OSCC patients (Table 3). The predictive value of SPRR3 towards OSCC prognosis was confirmed by K-M survival analysis based on TCGA database (log rank Pvalue $<0.05$; Fig. 5F).

\subsection{Low SPRR3 expression is correlated with carcinogenesis and progression of OSCC}

According to the OSCC patient data in the TCGA database, SPRR3 expression levels were significantly lower than those in oral normal tissues (P-value $<0.0001$, Figure $6 \mathrm{~A})$. ROC analysis was then performed to confirm the statistical relevance of these results. For this, AUC was calculated to identify the diagnostic specificity and sensitivity of SPRR3 expression. Based on the GSE30784 dataset and the TCGA database, down regulated SPRR3 levels yielded an AUC of 0.920 and 0.731 , respectively (Figs. 6B-C). In this regard, SPRR3 had positive diagnostic performance for OSCC patients' discrimination. Thus, SPRR3 could act as a potential diagnostic indicator for OSCC.

354 Student's t-test (two-tailed) and one-way ANOVA were thus performed and showed that low 355 SPRR3 expression was in the subgroups of patients with high alcohol consumption, poor 356 differentiation, non N0 staging, positive lymphovascular invasion, and positive perineural invasion 357 (Figs. 6D-H). The $\chi^{2}$ test was also utilized to figure out the correlation between SPRR3 expression 
358 of and the clinicopathological characteristics of OSCC. Consistent with previous tests, SPRR3

359 expression was significantly correlated with alcohol consumption $(\mathrm{P}-\mathrm{value}=0.011)$, histologic

360 grade $(\mathrm{P}$-value $<0.001)$, $\mathrm{N}$ staging $(\mathrm{P}$-value $=0.005)$, lymphovascular invasion $(\mathrm{P}$-value $=0.026)$

361 and perineural invasion $(\mathrm{P}-\mathrm{value}=0.016)($ Table 4$)$. These results reiterated that SPRR3 may act

362 as a factor related to tumor progression and/or metastasis.

363

$364 \quad 3.7$ Differential detection of SPRR3 protein in OSCC

365

366

367

368

369

370

371

372

373

374

375

376

377

378

379

380

381

382

383

384

385

386

387

388

389

390

IHC staining suggests that the SPRR3 protein levels in OSCC tissues vary according to the histologic grade of the cancer (Figs. 6I-L). Specifically, SPRR3 levels were lower in tumor tissues from patients at higher histologic grade when compared to those at lower grade.

\subsection{SPRR3 is enriched in various metabolic pathways and negatively associated with OSCC malignant progression}

To probe SPRR3-related pathways in an unbiased manner, GSEA analysis was conducted using OSCC data from TCGA. Based on the hallmark signature, SPRR3 was enriched in (i) apical surface, (ii) fatty acid metabolism, (iii) K-Ras signaling down and (iv) xenobiotic metabolism. GO-BP enrichment showed that SPRR3 was also correlated with (i) alcohol metabolic process, (ii) drug metabolic process, (iii) epidermis development and (iv) fatty acid derivative metabolic process. Lastly, KEGG enrichment assigned SPRR3 into (i) drug metabolism cytochrome P450, (ii) glycolysis/gluconeogenesis, (iii) metabolism of xenobiotic by cytochrome P450, (iv) retinol metabolism and (v) VEGF signaling pathway (Figs. 7A-C).

To further verify the role of SPRR3 in OSCC carcinogenesis and development, we analyzed the relationship between SPRR3 and other genes from the two module clusters previously screened, using the GSE30784 and TCGA-OSCC datasets. Interestingly, SPRR3 was positively correlated with genes from the module cluster 1 (CSTA, DSG1, EVPL, IVL, PPL, TGM1). CSTA has been reported as a potential OSCC biomarker (Hsiao et al., 2017). In contrast, SPRR3 was negatively correlated with a number of DEGs from the module cluster 2, including PLAU (Plasminogen Activator, Urokinase), COL5A3 (Collagen Type V Alpha 3 Chain), SERPINH1 (Serpin Family H Member 1), MMP 13, MMP7, COL4A6 (Collagen Type IV Alpha 6 Chain), and COL3A1 (Collagen Type III Alpha 1 Chain). Coincidentally, PLAU has been reported as an important factor involved in the regulation of cell migration, proliferation and invasion in OSCC (Zou et al., 2019), 
391 while MMP7 appears to have an oncogenic role in OSCC invasion and metastasis (Dasgupta et 392 al., 2012).

393 By accessing the GSE30784 and TCGA-OSCC datasets, we also examined major SPRR3 co394 expressing genes, including potential markers, which were closely associated with metabolic

395

396

397

398

399

400

401

402

403

404

405

406

407

408

409

410

411

412

413

414

415

416

417

418

419

420

421

422

423

424

425

processes and cancer progression. As a result, we observed that SPRR3 was positively correlated with some metabolic process markers including ADH1C (Alcohol Dehydrogenase 1C), ADH7 (Alcohol Dehydrogenase 7), ALDH1A1 (Aldehyde Dehydrogenase 1 Family Member A1), ALDH1A3 (Aldehyde Dehydrogenase 1 Family Member A3), CYP2E1 (Cytochrome P450 Family 2 Subfamily E Member 1). Among the EMT- and metastasis-related markers, we have also verified that SPRR3 was positively correlated with CDH1 (Cadherin 1) and TJP1 (Tight Junction Protein 1). Moreover, SPRR3 was negatively associated with CDH2 (Cadherin 2), FN1 (Fibronectin 1), MMP1, MMP9, SNAI1 (Snail Family Transcriptional Repressor 1), SNAI2 (Snail Family Transcriptional Repressor 2), TGFBI (Transforming Growth Factor Beta Induced) and VIM (Vimentin) (Fig. 7D for GSE30784, Fig. 7E for TCGA). These results indicated that SPRR3 could be involved in a series of metabolic processes, and the downregulation of SPRR3 may be potentially linked to the malignant progression of OSCC.

\section{Discussion}

OSCC, a subset of HNSCC, is one of the most common malignancies from the oral cavity worldwide. It is an aggressive tumor that bears a poor prognosis. In fact, OSCC patients at advanced stages, even after intensive treatment, may develop relapses and distant metastases. Therefore, early diagnosis and intervention remain critical for the management of OSCC. In this regard, measuring tumor markers has gradually become an important method for detecting the tumor status and monitoring therapeutic effects in the clinic.

The development and increasing use of tumor biomarkers has become a pivotal part of cancer diagnosis. Likewise, seeking novel tumor-related markers has provided new trains of thought in OSCC treatment (Csosz et al., 2017). Potential biomarkers with high specificity and sensitivity, identified in datasets by distinct Bioinformatic methods, have been continuously explored (Sun et al., 2015). Previous studies have determined a number of target genes by filtering DEGs in datasets (B. Yang et al., 2017; Zhao et al., 2018). Still, given the differences in specimens and database platforms, potential markers have failed to bear a significant clinical application value, so their verification process have been still far from clinical practice. Hence, we expect that DEGs obtained from biopsy-based sequencing, combined with the validation by independent public datasets and tissues analyses, will be more beneficial for the clinical diagnosis and prognosis of the disease. In

Peer] reviewing PDF | (2019:12:44257:2:2:NEW 21 May 2020) 
426 this study, we have focused on the identification of potential biomarkers which may possess 427 diagnostic and prognostic significance.

428 Current RNA-seq data has revealed 229 OSCC-related DEGs that were particularly enriched in 429 processes associated with tumorigenesis and malignant progression. By further screening gene 430 modules in a PPI network, a module cluster containing eight down regulated hub genes in OSCC 431 (cluster 1) was selected as a target. The core gene in this module was retrieved by acquiring OS 432 information from different datasets. Univariate Cox regression, LASSO regression and 433 multivariant Cox regression analyses were successively conducted in order to screen the key genes that could independently predict OSCC prognosis, resulting in the identification of SPRR3 as a central hub gene. Thereafter, we conducted K-M survival analysis and further established ROC curves. As a predictive marker, SPRR3 has considerable clinical significance in OSCC diagnosis and prognosis. Based on clinical data, a low expression of SPRR3 was correlated with patients' alcohol consumption, histologic grade, lymph node metastasis, lymphatic vascular invasion, and perineural metastasis. GSEA and Pearson correlation analyses have been also conducted for gene annotation. Putting together, we concluded that SPRR3 is potentially associated with epidermis development, metabolic processes, and tumor progression.

SPRRs are located in an evolutionarily conserved genetic cluster, designated as the epidermal differentiation complex (EDC) in the chromosome 1q21. Epidermis development and epithelial cell differentiation are some of the basic functions of SPRR proteins (Cabral et al., 2001; Candi et progression of many cancers. In OSCC, over-expressed SPRR2A (Small Proline Rich Protein 2A) can impair distant metastasis of tongue squamous cell carcinoma cells in vivo (Fang et al., 2016). Down-regulation of SPRR3 expression has been reported in esophageal squamous cell carcinoma, esophageal adenocarcinoma, anal and gastric cancers, while it has been strongly upregulated in colorectal and breast cancers (Cho et al., 2010; Kim et al., 2012). Nevertheless, the role of SPRR3 in oral tissues has never been explored. According to $\chi^{2}$ tests, here we observed that low SPRR3 expression is associated with OSCC progression, lymphovascular invasion and perineural invasion. GSEA enrichment analysis showed that SPRR3 is also correlated with K-Ras and VEGF signaling, which are classic pathways in OSCC carcinogenesis and progression (Caulin et al., 2004; Siriwardena et al., 2018).

TJP1 and CDH1 are classic inhibitors of tumor invasion and metastasis (Li et al., 2016). The positive correlation between SPRR3 and these two target genes indicate that SPRR3 might be essential for cell adhesion. Furthermore, SPRR3 was negatively correlated with some mesenchymal markers, such as CDH2, TGFBI, FN1, VIM, MMPs, and transcriptional factors such as SNAI1, SNAI2 (Li et al., 2016). Therefore, SPRR3 appears to work as a modulating factor involved in the maintenance of oral epithelial tissues and also in EMT inhibition. These particular 
463 prognosis. However, the SPRR3's role in suppressing EMT, as well as its mechanism(s) of action 464 in OSCC, require more in-depth investigation.

465 GSEA enrichments analysis indicates that a series of metabolic processes are impacted by SPRR3. 466 This observation is consistent with other results we acquired by $\chi^{2}$ test, where alcohol consumption 467 is correlated with SPRR3 expression. Particularly, alcohol consumption is an important risk factor 468 for OSCC, and the individual's ability to metabolize alcoholic beverages somehow reflects on its 469 ability to control cancer. Accordingly, ethanol has been classified as a carcinogenic substance in 470 humans (Zygogianni et al., 2011). Additionally, SPRR3 may be involved in the metabolism of 471 other substances, including fatty acid, drugs, and xenobiotics. It has been reported that ADH, 472 ALDH, and CYP2E1 are indispensable in some metabolic processes (Jin et al., 2013). Thus. the 473 relationship between SPRR3 and ADH, ALDH, CYP2E1 can confirm, to some extent, the role of 474 SPPR3 in metabolic processes.

475 Altogether, our current results reflect the close relationship between SPRR3 and OSCC 476 carcinogenesis and development. In addition, this work provides novel insights for follow-up 477 studies, which should better dissect the impact of SPRR3 in OSCC, at the molecular and cellular 478 level.

\section{Conclusion}

In our research, the expression of SPRR3 was detected to be dysregulated in OSCC by means of RNA sequence, and further was verified by Bioinformatics methods. SPRR3 could be a potential bio-marker for identifying OSCC, and its under-expression could predict the prognosis of patients with OSCC. Above all, SPRR3 could be a qualified diagnostic/prognostic biomarker for OSCC.

\section{Reference}

Abdalla, Z., Walsh, T., Thakker, N., \& Ward, C. M. (2017). Loss of epithelial markers is an early event in oral dysplasia and is observed within the safety margin of dysplastic and T1 OSCC biopsies. PLoS One, 12(12), e0187449. doi:10.1371/journal.pone.0187449

Anders, S., Pyl, P. T., \& Huber, W. (2015). HTSeq--a Python framework to work with highthroughput sequencing data. Bioinformatics, 31(2), 166-169. doi:10.1093/bioinformatics/btu638 
495

496

497

498

499

500

501

502

503

504

505

506

507

508

509

510

511

512

513

514

515

516

517

518

519

520

521

522

523

524

525

526

527

528

529

530

531

532

533

Aravind Subramanian, P. T. (2005). Gene set enrichment analysis: A knowledge-based approach for interpreting genome-wide expression profiles. Proc Natl Acad Sci.

Bader, G. D., \& Hogue, C. W. (2003). An automated method for finding molecular complexes in large protein interaction networks. BMC Bioinformatics, 4, 2. doi:10.1186/1471-2105-4-2

Cabral, A., Voskamp, P., Cleton-Jansen, A. M., South, A., Nizetic, D., \& Backendorf, C. (2001). Structural organization and regulation of the small proline-rich family of cornified envelope precursors suggest a role in adaptive barrier function. Journal of Biological Chemistry, 276(22), 19231-19237. doi:10.1074/jbc.M100336200

Candi, E., Paci, M., Oddi, S., Paradisi, A., Guerrieri, P., \& Melino, G. (2000). Ordered structure acquisition by the $\mathrm{N}$ - and $\mathrm{C}$-terminal domains of the small proline-rich 3 protein. Journal of Cellular Biochemistry, 77(2), 179-185. doi:10.1002/(sici)10974644(20000501)77:2<179::Aid-jcb2>3.0.Co;2-h

Candi, E., Schmidt, R., \& Melino, G. (2005). The cornified envelope: A model of cell death in the skin. Nature Reviews Molecular Cell Biology, 6(4), 328-340. doi:10.1038/nrm1619

Caulin, C., Nguyen, T., Longley, M. A., Zhou, Z., Wang, X. J., \& Roop, D. R. (2004). Inducible activation of oncogenic K-ras results in tumor formation in the oral cavity. Cancer Res, 64(15), 5054-5058. doi:10.1158/0008-5472.CAN-04-1488

Chabanais, J., Labrousse, F., Chaunavel, A., Germot, A., \& Maftah, A. (2018). POFUT1 as a Promising Novel Biomarker of Colorectal Cancer. Cancers (Basel), 10(11). doi:10.3390/cancers10110411

Cho, D.-H., Jo, Y. K., Roh, S. A., Na, Y.-S., Kim, T. W., Jang, S. J., . . . Kim, J. C. (2010). Upregulation of SPRR3 Promotes Colorectal Tumorigenesis. Molecular Medicine, 16(78), 271-277. doi:10.2119/molmed.2009.00187

Csosz, E., Labiscsak, P., Kallo, G., Markus, B., Emri, M., Szabo, A., . . . Marton, I. (2017). Proteomics investigation of OSCC-specific salivary biomarkers in a Hungarian population highlights the importance of identification of population-tailored biomarkers. PLoS One, 12(5). doi:10.1371/journal.pone.0177282

Dasgupta, S., Dash, R., Das, S. K., Sarkar, D., \& Fisher, P. B. (2012). Emerging strategies for the early detection and prevention of head and neck squamous cell cancer. J Cell Physiol, 227(2), 467-473. doi:10.1002/jcp.22767

Fang, Z., Zhang, S., Wang, Y., Shen, S., Wang, F., Hao, Y., .. Y Yang, H. (2016). Long non-coding RNA MALAT-1 modulates metastatic potential of tongue squamous cell carcinomas partially through the regulation of small proline rich proteins. BMC Cancer, 16, 706. doi:10.1186/s12885-016-2735-x

Gautier, L., Cope, L., Bolstad, B. M., \& Irizarry, R. A. (2004). affy--analysis of Affymetrix GeneChip data at the probe level. Bioinformatics, 20(3), 307-315. doi:10.1093/bioinformatics/btg405

Hsiao, Y.-C., Chi, L.-M., Chien, K.-Y., Chiang, W.-F., Chen, S.-F., Chuang, Y.-N., . . Yu, J.-S. (2017). Development of a Multiplexed Assay for Oral Cancer Candidate Biomarkers Using

Peer] reviewing PDF | (2019:12:44257:2:2:NEW 21 May 2020) 
534

535

536

537

538

539

540

541

542

543

544

545

546

547

548

549

550

551

552

553

554

555

556

557

558

559

560

561

562

563

564

565

566

567

568

569

570

571

572

Peptide Immunoaffinity Enrichment and Targeted Mass Spectrometry. Molecular \& Cellular Proteomics, 16(10), 1829-1849. doi:10.1074/mcp.RA117.000147

Huang da, W., Sherman, B. T., \& Lempicki, R. A. (2009). Systematic and integrative analysis of large gene lists using DAVID bioinformatics resources. Nat Protoc, 4(1), 44-57. doi:10.1038/nprot.2008.211

Jin, M., Ande, A., Kumar, A., \& Kumar, S. (2013). Regulation of cytochrome P450 2e1 expression by ethanol: role of oxidative stress-mediated pkc/jnk/sp1 pathway. Cell Death Dis, 4, e554. doi:10.1038/cddis.2013.78

Kanehisa, M., Furumichi, M., Tanabe, M., Sato, Y., \& Morishima, K. (2017). KEGG: new perspectives on genomes, pathways, diseases and drugs. Nucleic Acids Res, 45(D1), D353D361. doi:10.1093/nar/gkw1092

Kim, J. C., Yu, J. H., Cho, Y. K., Jung, C. S., Ahn, S. H., Gong, G., . . . Cho, D.-H. (2012). Expression of SPRR3 is associated with tumor cell proliferation in less advanced stages of breast cancer. Breast Cancer Research and Treatment, 133(3), 909-916. doi:10.1007/s10549-011-1868-5

Li, H., Xu, F., Li, S., Zhong, A., Meng, X., \& Lai, M. (2016). The tumor microenvironment: An irreplaceable element of tumor budding and epithelial-mesenchymal transition-mediated cancer metastasis. Cell Adh Migr, 10(4), 434-446. doi:10.1080/19336918.2015.1129481

Liu, W., Liu, J., \& Rajapakse, J. C. (2018). Gene Ontology Enrichment Improves Performances of Functional Similarity of Genes. Sci Rep, 8(1), 12100. doi:10.1038/s41598-018-304550

Lohavanichbutr, P., Mendez, E., Holsinger, F. C., Rue, T. C., Zhang, Y., Houck, J., .. . Chen, C. (2013). A 13-gene signature prognostic of HPV-negative OSCC: discovery and external validation. Clin Cancer Res, 19(5), 1197-1203. doi:10.1158/1078-0432.CCR-12-2647

Lu, H., Wu, B., Ma, G., Zheng, D., Song, R., Huang, E., . . Lu, B. (2017). Melatonin represses oral squamous cell carcinoma metastasis by inhibiting tumor-associated neutrophils. Am J Transl Res, 9(12), 5361-5374.

Matthews, H., Ranson, M., \& Kelso, M. J. (2011). Anti-tumour/metastasis effects of the potassium-sparing diuretic amiloride: an orally active anti-cancer drug waiting for its callof-duty? International Journal of Cancer, 129(9), 2051-2061. doi:10.1002/ijc.26156

Pertea, M., Kim, D., Pertea, G. M., Leek, J. T., \& Salzberg, S. L. (2016). Transcript-level expression analysis of RNA-seq experiments with HISAT, StringTie and Ballgown. Nat Protoc, 11(9), 1650-1667. doi:10.1038/nprot.2016.095

Shannon, P., Markiel, A., Ozier, O., Baliga, N. S., Wang, J. T., Ramage, D., . . Ideker, T. (2003). Cytoscape: a software environment for integrated models of biomolecular interaction networks. Genome Res, 13(11), 2498-2504. doi:10.1101/gr.1239303Shridhar, K., Walia, G. K., Aggarwal, A., Gulati, S., Geetha, A. V., Prabhakaran, D., . . Rajaraman, P. (2016). DNA methylation markers for oral pre-cancer progression: A critical review. Oral Oncology, 53, 1-9. doi:10.1016/j.oraloncology.2015.11.012

Peer) reviewing PDF | (2019:12:44257:2:2:NEW 21 May 2020) 
573 Siriwardena, S., Tsunematsu, T., Qi, G., Ishimaru, N., \& Kudo, Y. (2018). Invasion-Related

574

575

576

577

578

579

580

581

582

583

584

585

586

587

588

589

590

591

592

593

594

595

596

597

598

599

600

601

602

603

604

605

606

607

608

609

610

611 Factors as Potential Diagnostic and Therapeutic Targets in Oral Squamous Cell Carcinoma-A Review. Int J Mol Sci, 19(5). doi:10.3390/ijms19051462

Specht, S., Isse, K., Nozaki, I., Lunz, J. G., III, \& Demetris, A. J. (2013). SPRR2A expression in cholangiocarcinoma increases local tumor invasiveness but prevents metastasis. Clinical \& Experimental Metastasis, 30(7), 877-890. doi:10.1007/s10585-013-9589-2

Subramanian, A., Tamayo, P., Mootha, V. K., Mukherjee, S., Ebert, B. L., Gillette, M. A., . . . Mesirov, J. P. (2005). Gene set enrichment analysis: a knowledge-based approach for interpreting genome-wide expression profiles. Proceedings of the National Academy of Sciences of the United States of America, 102(43), 15545-15550. doi:10.1073/pnas.0506580102

Sun, T.-t., Du, W., Chen, H. Y., Hong, J., \& Fang, J. Y. (2015). TMEFF2 Deregulation Contributes to Gastric Carcinogenesis and Indicates Poor Survival Outcome. Clinical Gastroenterology and Hepatology, 13(7), E79-E79. doi:10.1016/j.cgh.2015.04.055

Szklarczyk, D., Franceschini, A., Kuhn, M., Simonovic, M., Roth, A., Minguez, P., . . . von Mering, C. (2011). The STRING database in 2011: functional interaction networks of proteins, globally integrated and scored. Nucleic Acids Research, 39, D561-D568. doi:10.1093/nar/gkq973

Szklarczyk, D., Morris, J. H., Cook, H., Kuhn, M., Wyder, S., Simonovic, M., . . von Mering, C. (2017). The STRING database in 2017: quality-controlled protein-protein association networks, made broadly accessible. Nucleic Acids Research, 45(D1), D362-D368. doi:10.1093/nar/gkw937

Tang, Z., Li, C., Kang, B., Gao, G., Li, C., \& Zhang, Z. (2017). GEPIA: a web server for cancer and normal gene expression profiling and interactive analyses. Nucleic Acids Research, 45(W1), W98-W102. doi:10.1093/nar/gkx247

Tomczak, K., Czerwinska, P., \& Wiznerowicz, M. (2015). The Cancer Genome Atlas (TCGA): an immeasurable source of knowledge. Contemp Oncol (Pozn), 19(1A), A68-77. doi:10.5114/wo.2014.47136

Toruner, G. A., Ulger, C., Alkan, M., Galante, A. T., Rinaggio, J., Wilk, R., . . Dermody, J. J. (2004). Association between gene expression profile and tumor invasion in oral squamous cell carcinoma. Cancer Genetics and Cytogenetics, 154(1), 27-35. doi:10.1016/j.cancergencyto.2004.01.026

Wang, L., Feng, Z., Wang, X., Wang, X., \& Zhang, X. (2010). DEGseq: an R package for identifying differentially expressed genes from RNA-seq data. Bioinformatics, 26(1), 136138. doi:10.1093/bioinformatics/btp612

Yang, B., Chen, Z., Huang, Y., Han, G., \& Li, W. (2017). Identification of potential biomarkers and analysis of prognostic values in head and neck squamous cell carcinoma by bioinformatics analysis. Oncotargets and Therapy, 10, 2315-2321. doi:10.2147/ott.S135514

Peer) reviewing PDF | (2019:12:44257:2:2:NEW 21 May 2020) 
612 Yang, Z., Liang, X., Fu, Y., Liu, Y., Zheng, L., Liu, F., . . Xu, X. (2019). Identification of AUNIP

613 as a candidate diagnostic and prognostic biomarker for oral squamous cell carcinoma.

$614 \quad$ EBioMedicine, 47, 44-57. doi:10.1016/j.ebiom.2019.08.013

615 Yu, G., Wang, L.-G., Han, Y., \& He, Q.-Y. (2012). clusterProfiler: an R Package for Comparing

616 Biological Themes Among Gene Clusters. Omics-a Journal of Integrative Biology, 16(5),

617 284-287. doi:10.1089/omi.2011.0118

618 Zanoni, D. K., Montero, P. H., Migliacci, J. C., Shah, J. P., Wong, R. J., Ganly, I., \& Patel, S. G.

619 (2019). Survival outcomes after treatment of cancer of the oral cavity (1985-2015). Oral

620 Oncology, 90, 115-121. doi:10.1016/j.oraloncology.2019.02.001

621 Zhang, S., Cao, R., Li, Q., Yao, M., Chen, Y., \& Zhou, H. (2019). Comprehensive analysis of

622 lncRNA-associated competing endogenous RNA network in tongue squamous cell

623 carcinoma. PeerJ, 7, e6397. doi:10.7717/peerj.6397

624 Zhao, X., Sun, S., Zeng, X., \& Cui, L. (2018). Expression profiles analysis identifies a novel three-

625 mRNA signature to predict overall survival in oral squamous cell carcinoma. American

626 Journal of Cancer Research, 8(3), 450-461.

627 Zou, B., Li, J., Xu, K., Liu, J.-L., Yuan, D.-Y., Meng, Z., \& Zhang, B. (2019). Identification of

628 key candidate genes and pathways in oral squamous cell carcinoma by integrated

629 Bioinformatics analysis. Experimental and Therapeutic Medicine, 17(5), 4089-4099.

$630 \quad$ doi:10.3892/etm.2019.7442

631 Zygogianni, A. G., Kyrgias, G., Karakitsos, P., Psyrri, A., Kouvaris, J., Kelekis, N., \& Kouloulias,

632

633 V. (2011). Oral squamous cell cancer: early detection and the role of alcohol and smoking. Head Neck Oncol, 3, 2. doi:10.1186/1758-3284-3-2

634

6357 Data Availability Statement

636

637 All datasets [GENERATED/ANALYZED] for this study are included in the manuscript and the 638 supplementary files.

639 
Figure 1

The workflow of this study.

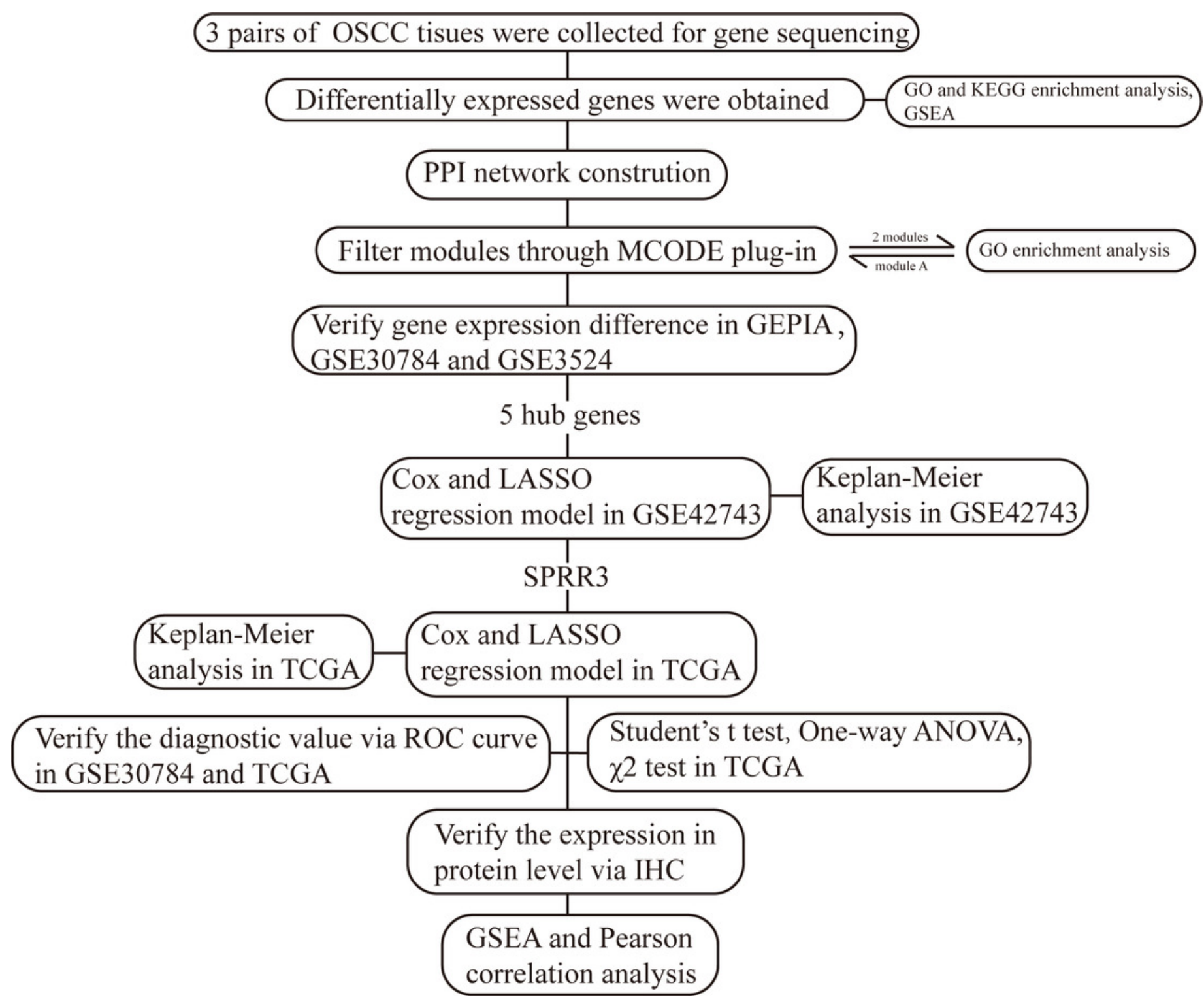




\section{Figure 2}

DEGs between OSCC and adjacent tissues.

A. Volcano plot exhibits the high-throughput RNA-seq result. Among them, 85 genes were up-regulated (red plots) and 144 were down-regulated (green plots). SPRR3 was marked in the figure. B. Heatmap of RNA sequencing result, red for up-regulated genes and blue for down-regulated genes. SPRR3 was marked in the figure. C. GO enrichment of the DEGs. The DEGs are mostly correlated with keratinization, keratinocyte differentiation, ECM organization, cell adhesion and migration. D. KEGG enrichment of the DEGs. The DEGs were enriched in xenobiotic metabolism, drug metabolism and chemical carcinogenesis, etc. E. GSEA in hallmark enrichment, NES, P-value and FDR were listed respectively. The DEGs were enriched in epithelial mesenchymal transition, K-Ras signaling down and TGF- $\beta$ signaling, etc.

F. GSEA in GO enrichment. The DEGs were enriched in DNA repication, epidermal cell differentiation, ECM disassembly and positve regulation of cell activation, etc. G. GSEA in KEGG enrichment. The DEGs were enriched in drug metabolism, xenobiotic metabolism, focal adhesion, P53 signaling and tight junction, etc. 
A

B
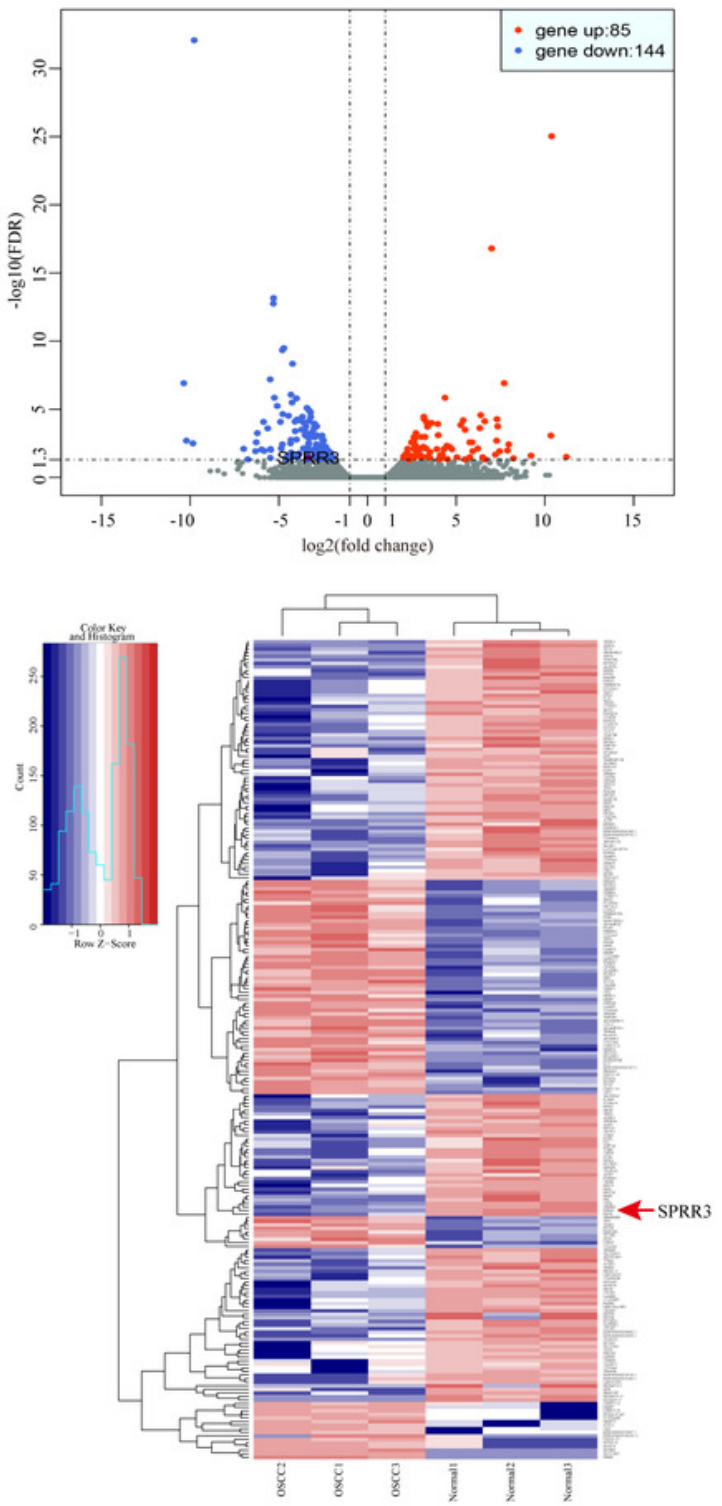

C

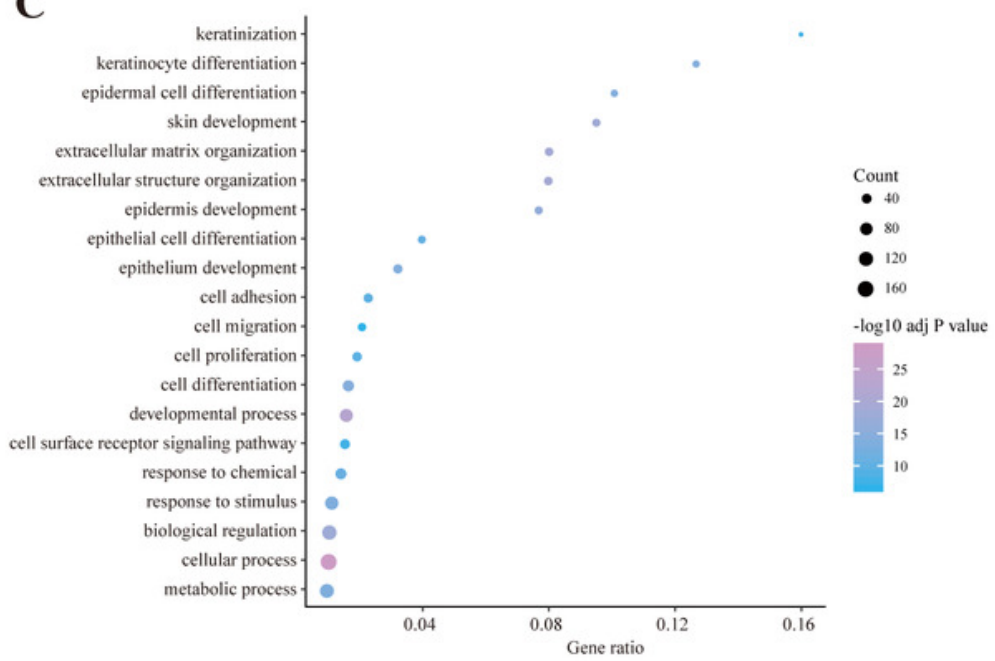

D

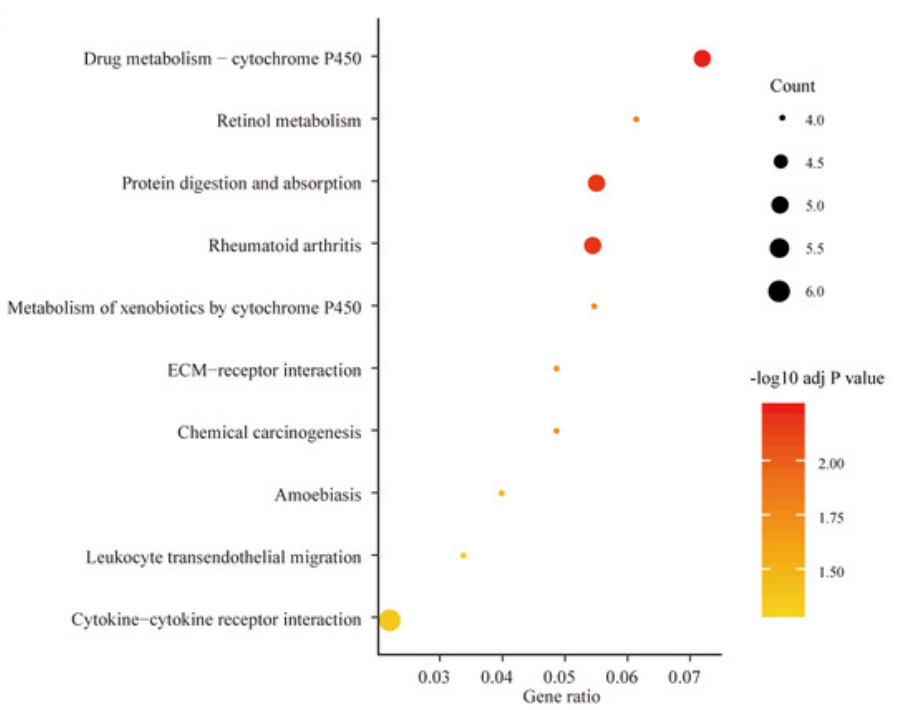

E

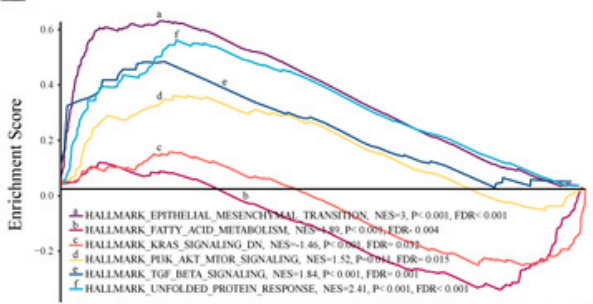

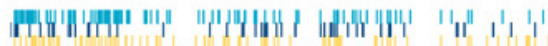

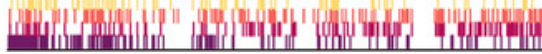

F

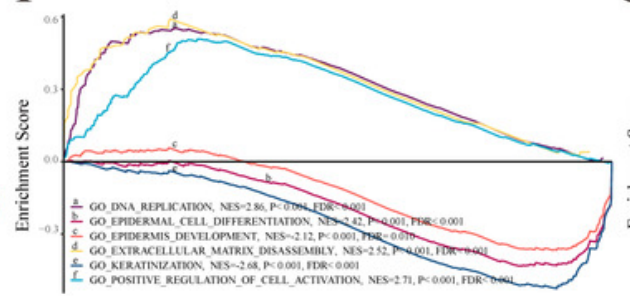

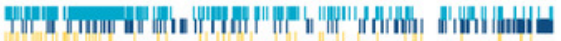

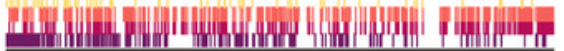

G

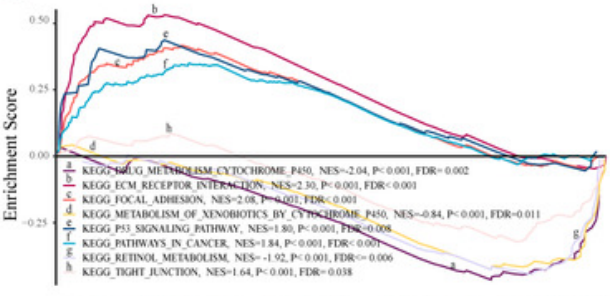

Fin'

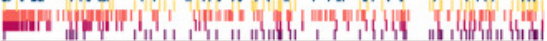




\section{Figure 3}

Selected core modules of PPI network and functional enrichment analysis.

A. Genes in module cluster 1 (blue nodes) were down-regulated in OSCC tissues. B. Genes in module cluster 2 (red nodes) were up-regulated in OSCC. C. GO enrichment shows that module cluster 1 was correlated with keratinization, cornification and epidermal cell differentiation in BP. D. Genes in molecular cluster 1 were enriched in cornified envelop, desmosome and cell junction etc. in CC. E. Genes in molecular cluster 1 were enriched in protein binding and bridging in MF. The size of the dot refers to the gene counts enriched in that term, and the color refers to the FDR according to the FDR gauge. F. Module cluster 2 was mostly enriched in collagen metabolic process and extracellular structure organization in BP. G. Genes in molecular cluster 1 were enriched in extracellular matrix in CC. H. Genes in molecular cluster 1 were enriched in collagen binding in MF. 
A

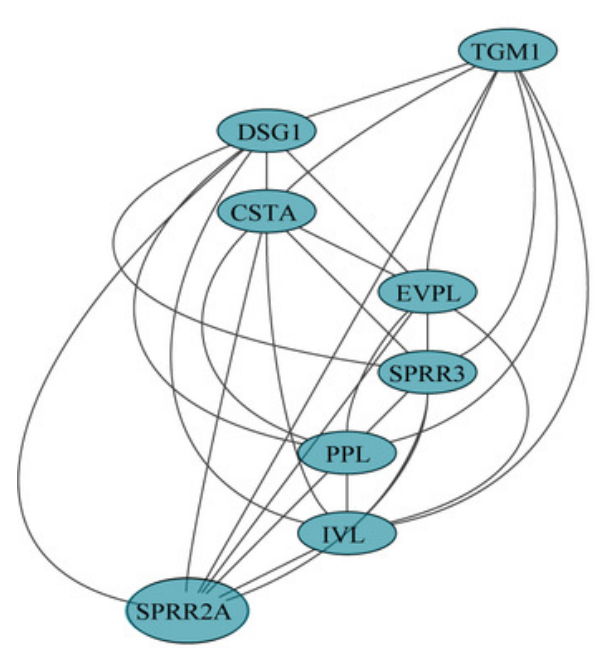

C

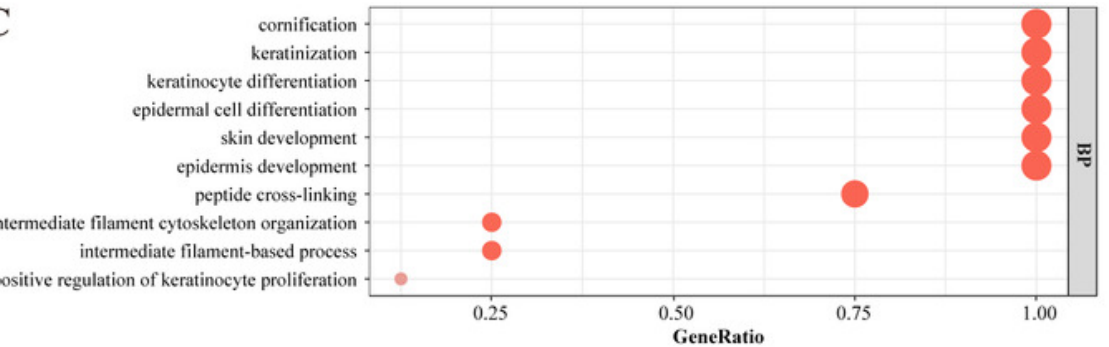

D

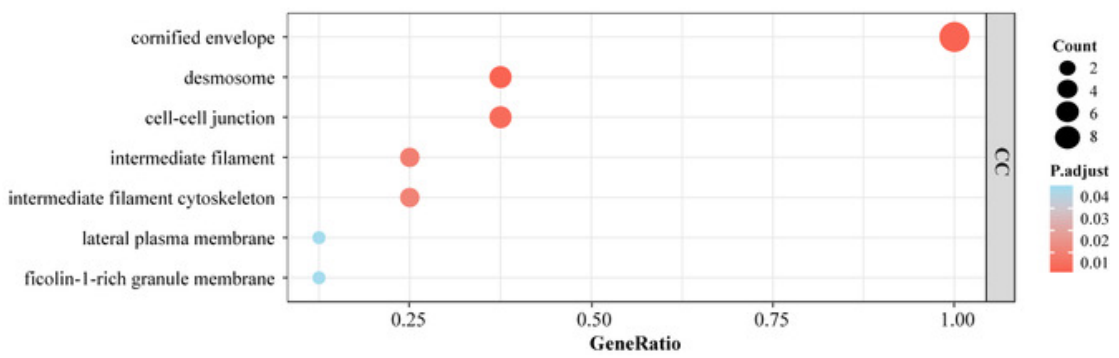

$\mathbf{E}$
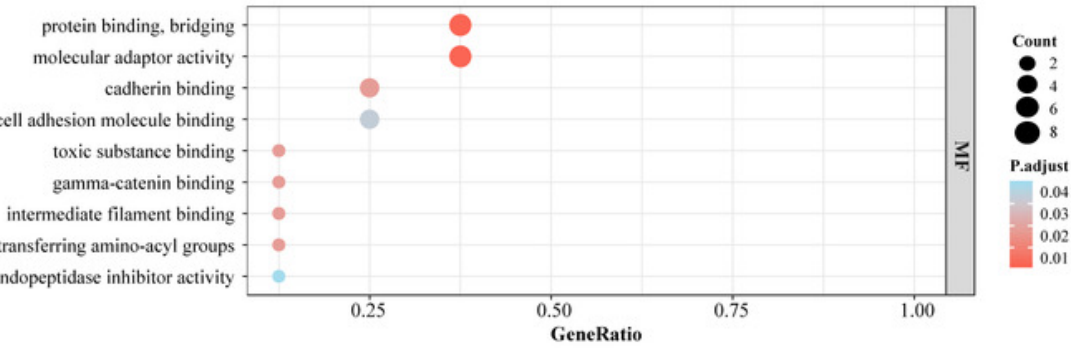

B

F
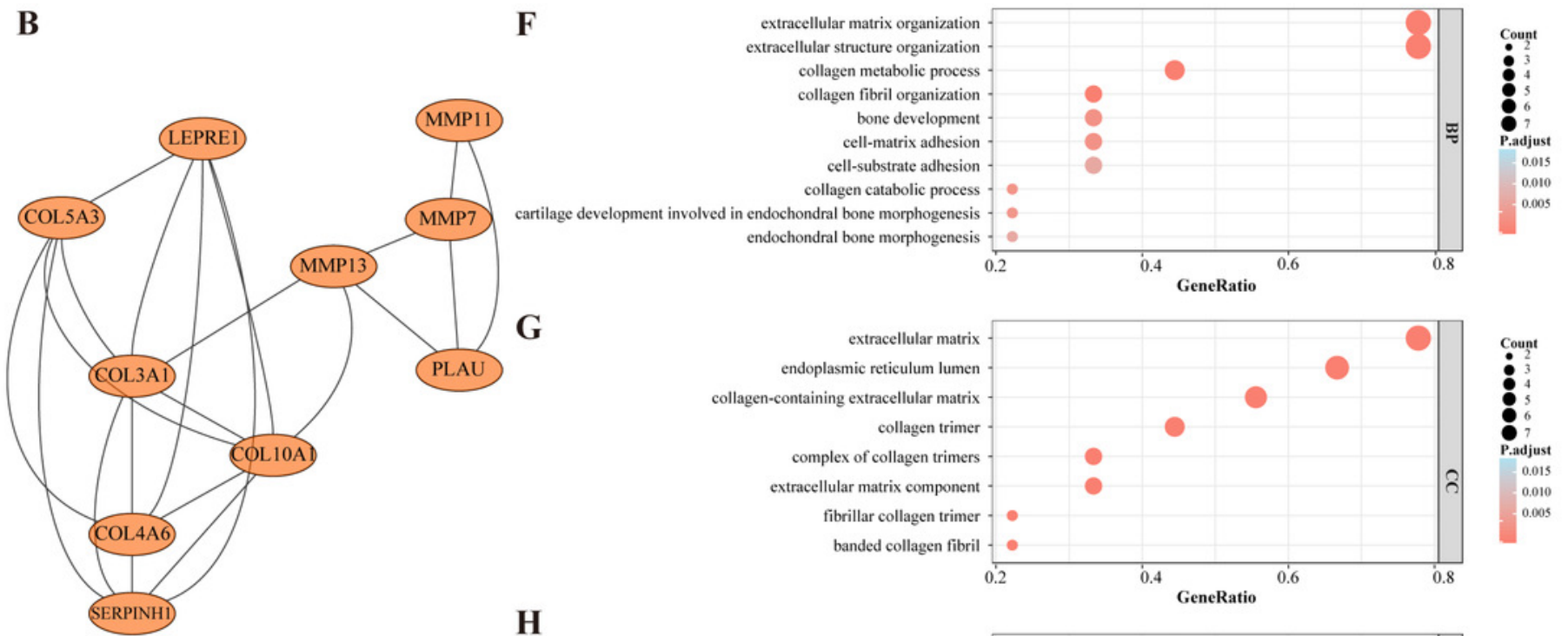

H
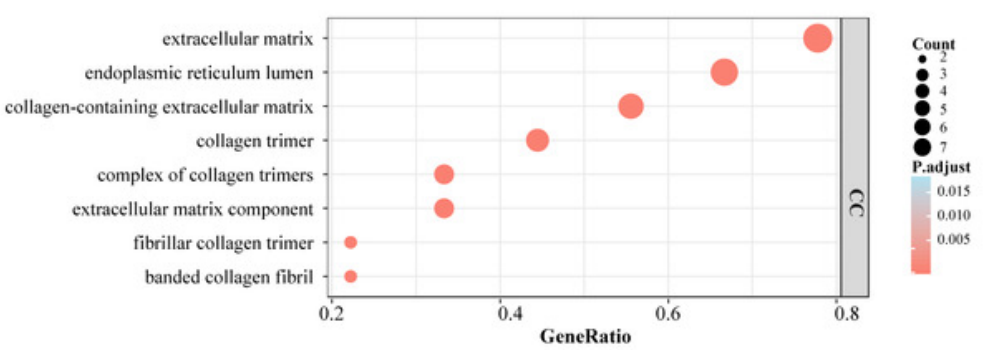

extracellular matrix structural constituent conferring tensile strength collagen binding collagen binding
extracellular matrix structural constituent serine-type endopeptidase activity serine-type peptidase activity serine hydrolase activity metalloendopeptidase activity heparin binding metallopeptidase activity glycosaminoglycan binding

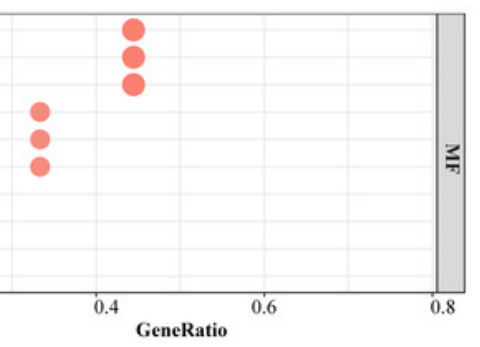




\section{Figure 4}

Transcriptional level of DEGs in OSCC.

A-H . The transcription level of DEGs in module cluster 1 according to GEPIA. The expression levels of CSTA, PPL, EVPL, SPRR3 and TGM1 with P-value $<0.05$ in student's t test (two tailed) were significantly differentially expressed between tumor and normal tissues in HNSCC (n.s represents not significant). I-M. The differentially transcript level of candidate genes in module cluster 1 in GSE30784 dataset. The expression levels of CSTA, PPL, EVPL, SPRR3 and TGM1 with P-value $<0.001$ were significantly differentially expressed between tumor and normal tissues in OSCC by student's t test (two tailed). 
A
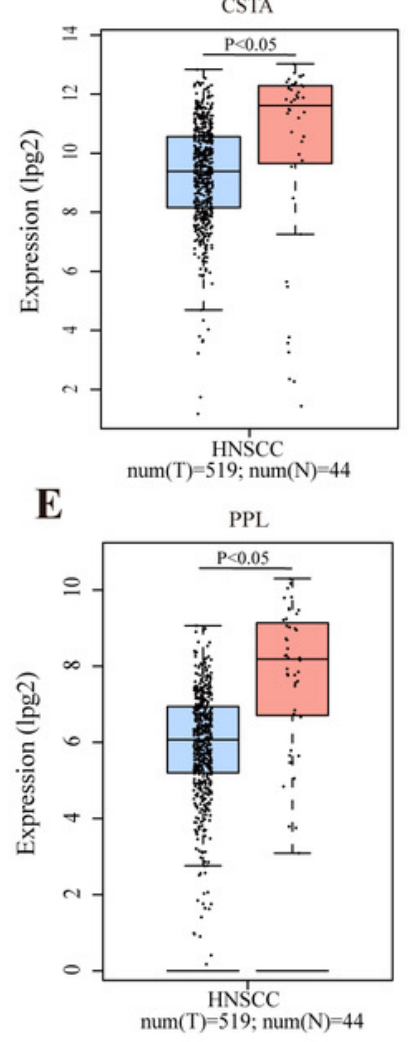

I

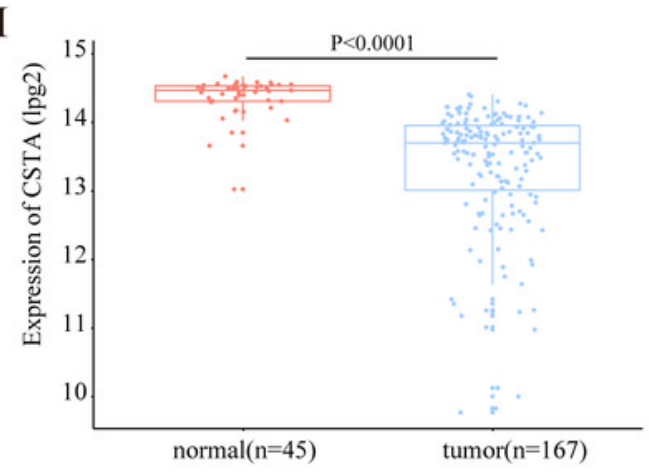

C
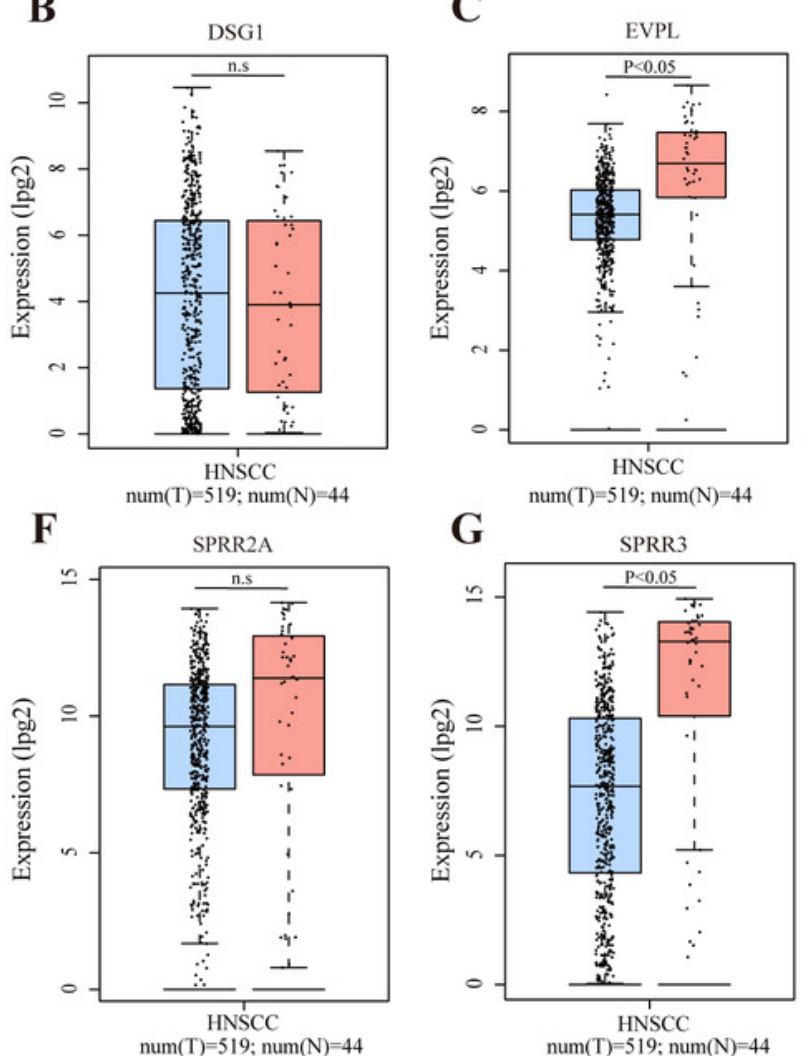
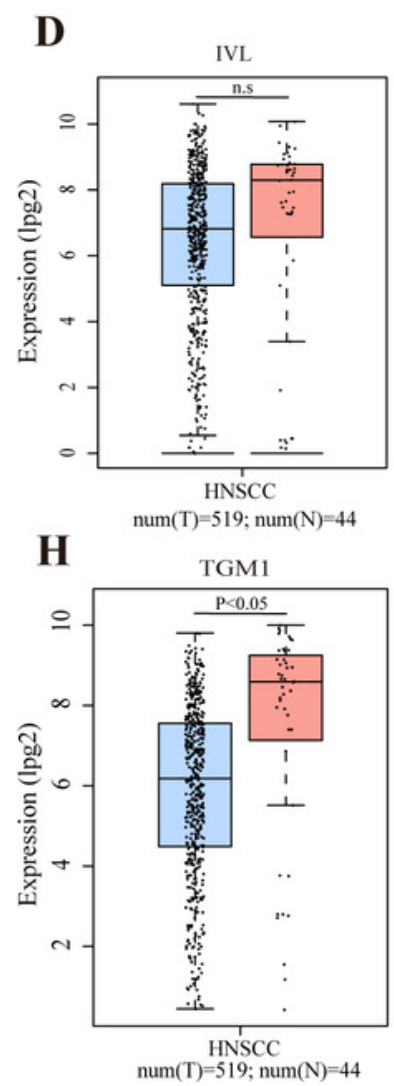

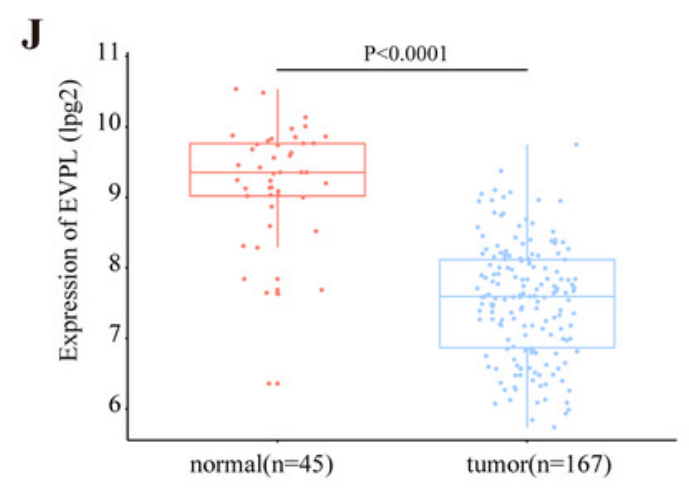

K

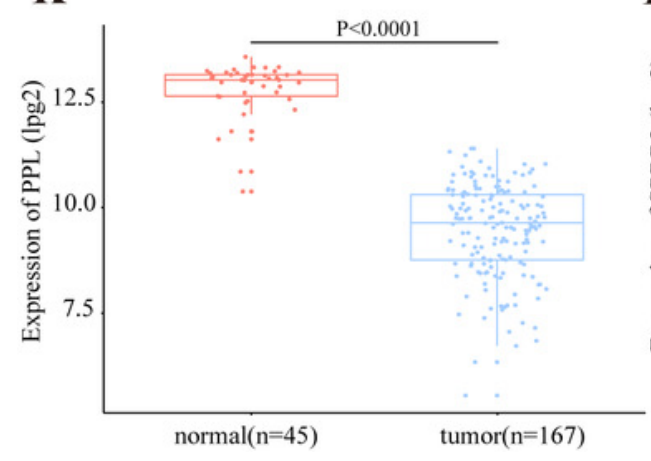

L

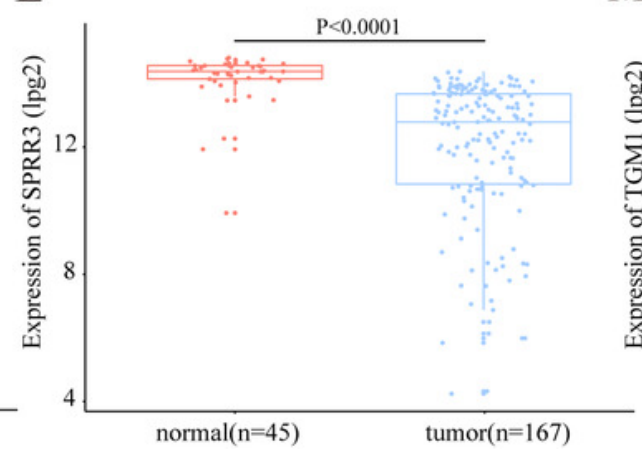

M

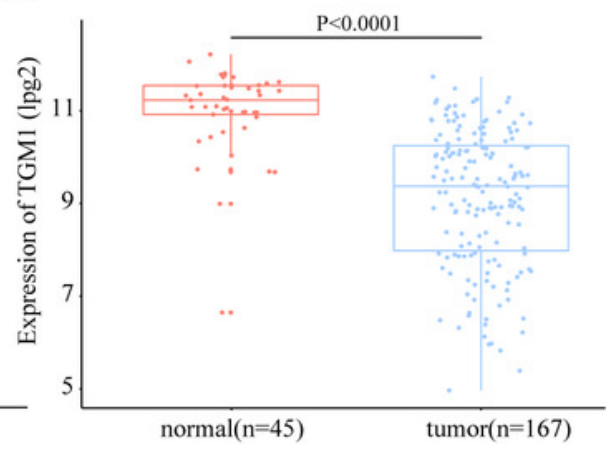


Figure 5

Prognostic significance.

A. Validation was performed for tuning parameter selection through the LASSO regression analysis in GSE42743. LASSO, the least absolute shrinkage and selection operator. B.

Elaboration for LASSO coefficient profiles of prognostic RNAs . C. K-M survival analysis based on GSE42743 dataset according to SPRR3 expression level. D. Validation was performed for tuning parameter selection through the LASSO regression model in TCGA. E. Elaboration for LASSO coefficient profiles of prognostic RNAs. F. K-M survival analysis based on TCGA-OSCC dataset according to SPRR3 expression level.

A

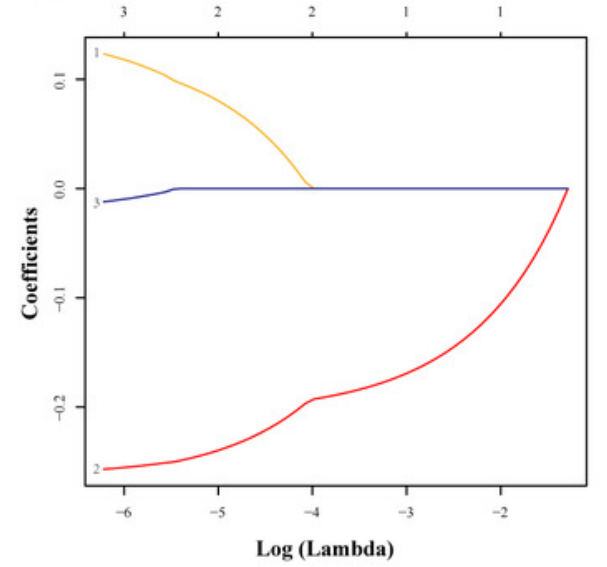

D

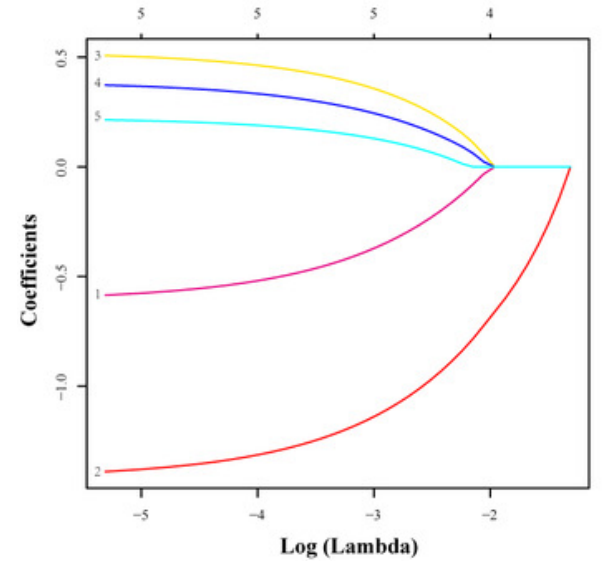

B

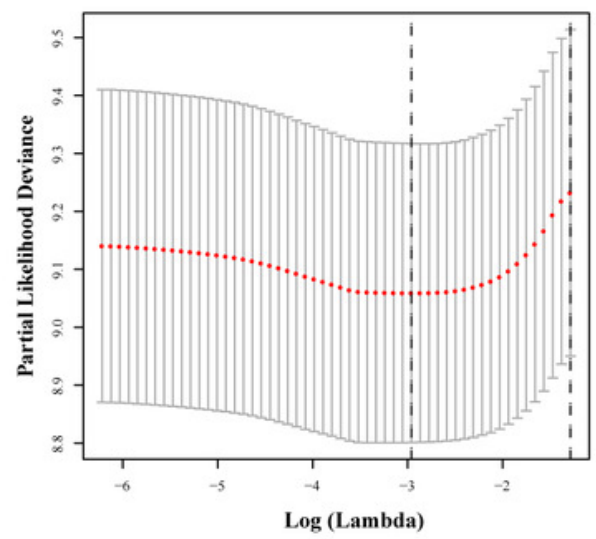

E

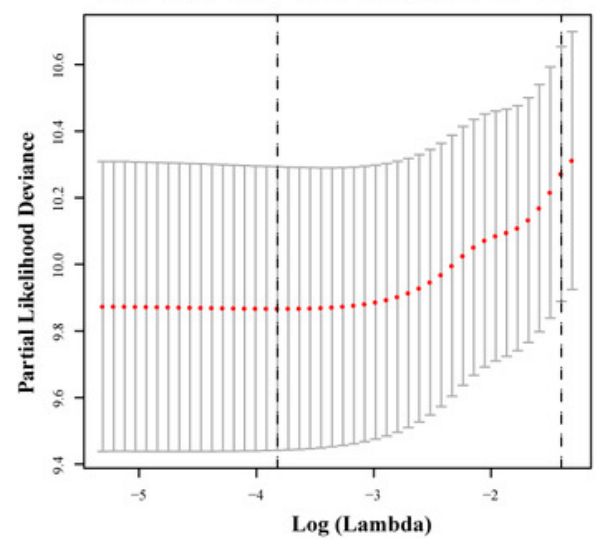

C

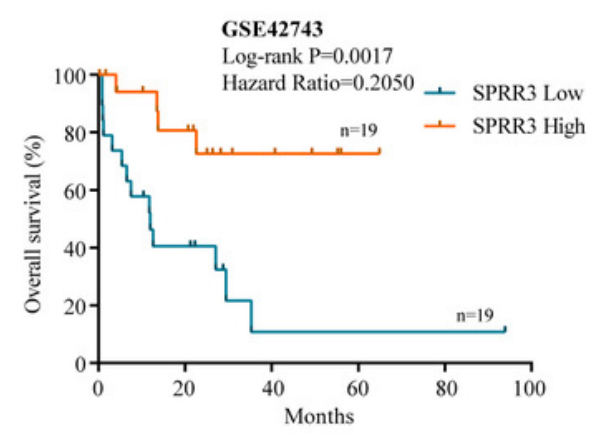

F

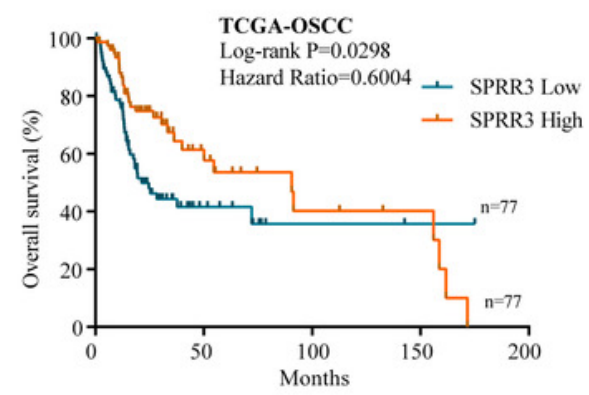




\section{Figure 6}

Clinical correlation of SPRR3 expression level in OSCC.

A. The expression of SPRR3 in TCGA-OSCC database, the expression level of SPRR3 was significantly lower in tumor group than that in normal group by student's t test (two tailed).

B. In GSE30784, ROC curve was created to verify the diagnostic value of SPRR3. AUC = 0.920, P-value < 0.001. C. In ROC curve analysis from TCGA database, AUC was 0.731 and Pvalue $=0.001$. D-H. Two-tailed student's t test and one-way ANOVA test suggested that SPRR3 was differentially expressed in groups in accordance of whether the patients had alcohol consumption $(P=0.0139)$, the histological grade ( $P$-value $<0.0001)$, $N$ stage ( $P$-value $=0.0076)$ of the patients, and whether the patients had lymphovascular invasion (P-value $=$ $0.0102)$, perineural invasion (P-value $=0.0068)$ respectively. $\mathbf{I}$. Protein expression level of SPRR3 in well differentiated OSCC tissue. J. Protein expression level of SPRR3 in moderate differentiated OSCC tissue. K. Protein expression level of SPRR3 in poor differentiated OSCC tissue. L. Protein expression level of SPRR3 in adjacent tissue. The image on the left in the group was taken at the low power field $(100 *)$, the right was taken at the high-power field (400*). The black line represents the length of $100 \mathrm{~nm}$. 
A

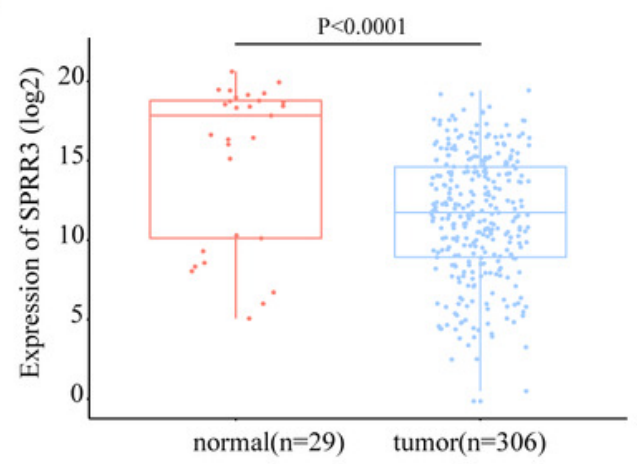

D

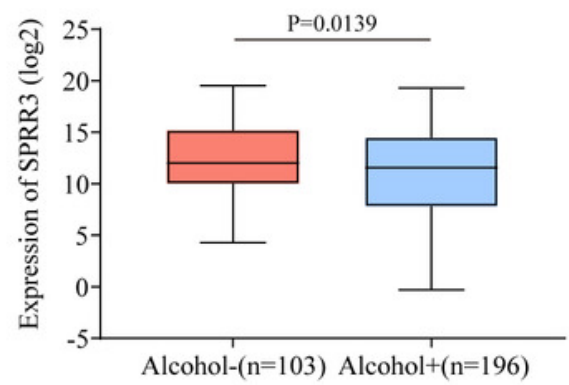

F

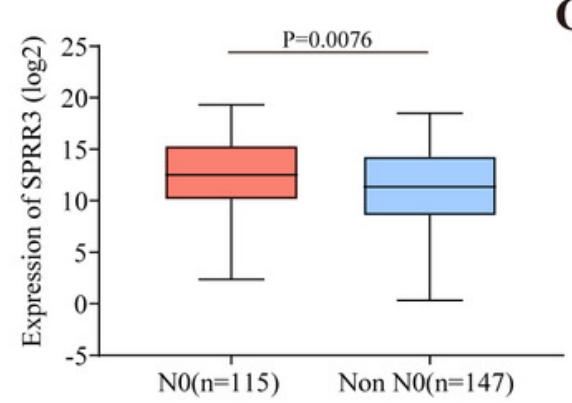

I

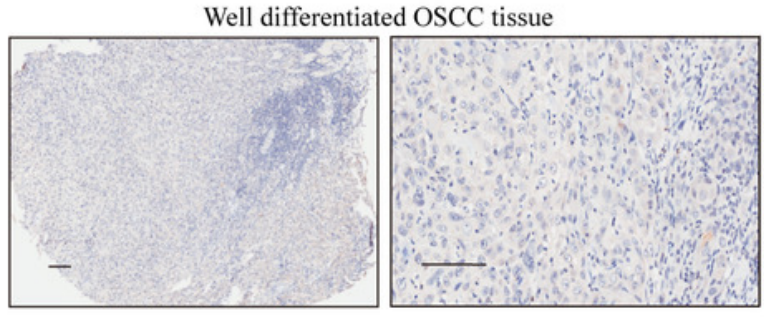

K

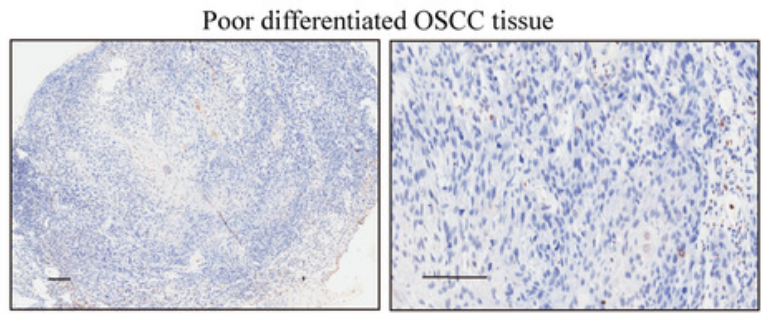

G
B

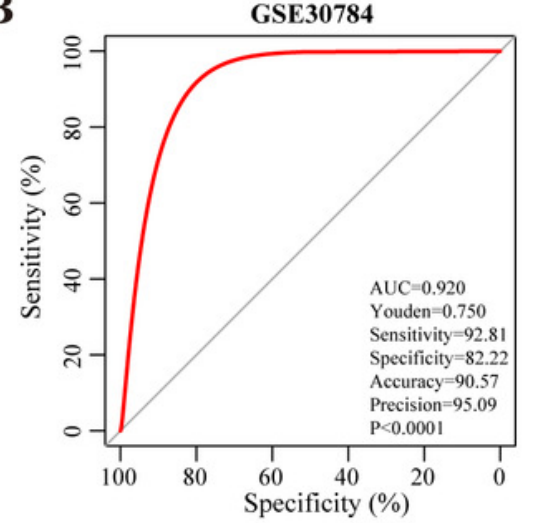

C

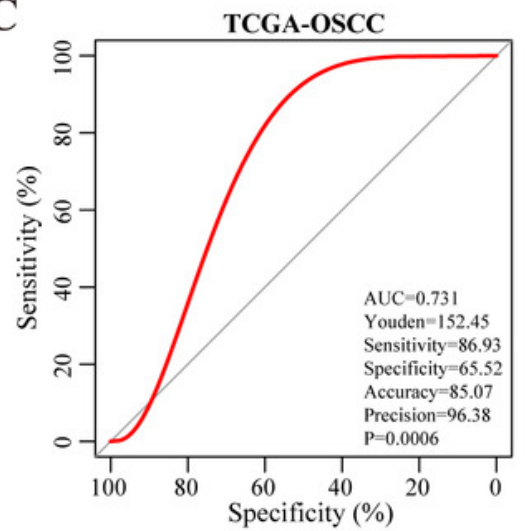

E
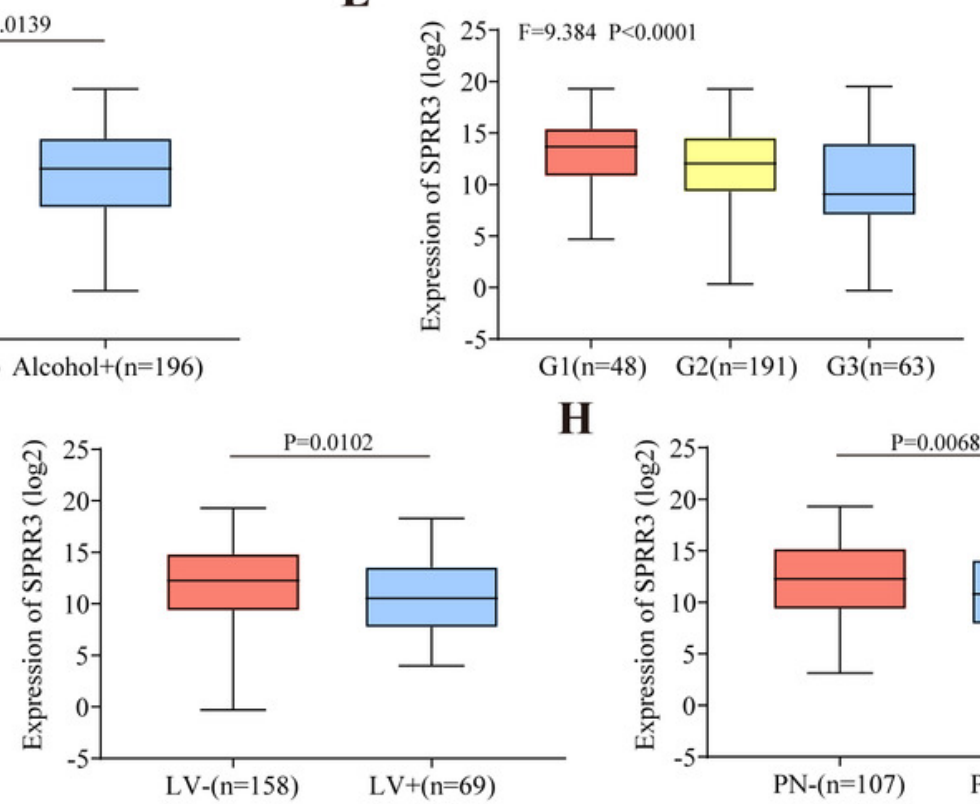

H

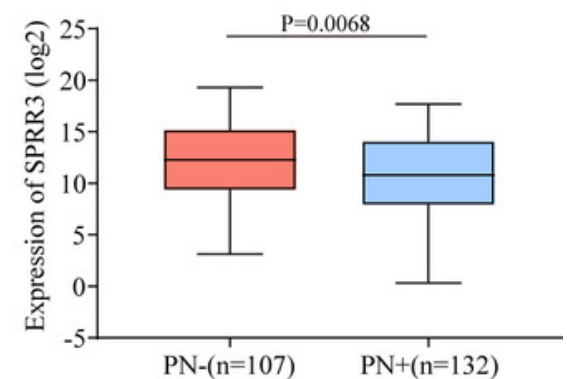

J

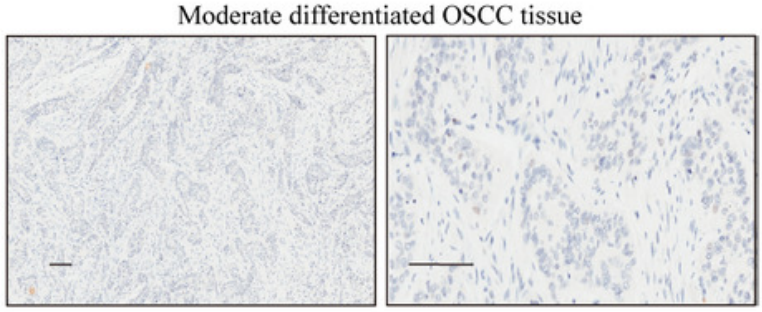

L

Adjcacent oral tissue

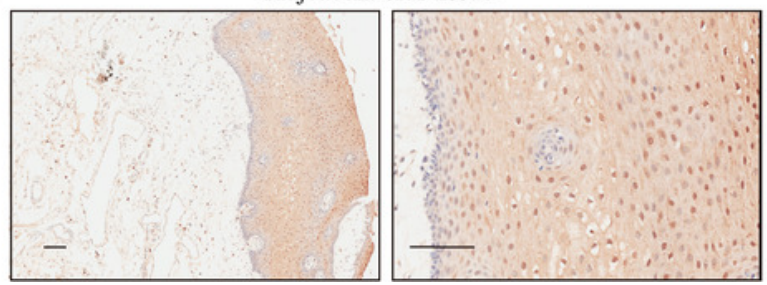




\section{Figure 7}

GSEA analysis of SPRR3 in TCGA database.

A. SPRR3 was enriched in apical surface, fatty acid metabolism, K-Ras signaling down and xenobiotic metabolism in hallmark terms B. SPRR3 was enriched in alcohol metabolic, drug metabolic, epidermis development, fatty acid derivative process and response to xenobiotic stimulus in GO terms. C. SPRR3 was enriched in drug metabolism cytochrome P450, fatty acid metabolism, glycolysis gluconeogenesis, metabolism of xenobiotics by cytochrome and VEGF signaling pathway in KEGG terms. D. Pearson correlation analysis between DEGs in GSE30784 dataset. SPRR3 was positively correlated with other DEGs in module cluster 1 (CSTA, DSG1, EVPL, IVL, PPL, TGM1) and negatively correlated with DEGs in Module cluster 2 (PLAU, COL10A1, COL3A1, COL4A6, COL5A3), and it was positively correlated with ADH1C, ADH7, ALDH1A1, ALDH1A3, ALDH3B2, CYP2E1, CDH1, TJP1, it was negatively correlated with CDH2, FN1, SNAI1, SNAI2 and TGFBI. E. Pearson correlation analysis between DEGs in TCGAOSCC dataset. SPRR3 was positively correlated with other DEGs in module cluster 1 and negatively correlated with DEGs in Module cluster 2 , and it was positively correlated with ADH1C, ADH7, ALDH1A1, ALDH1A3, ALDH3B2, CYP2E1, CDH1, TJP1, it was negatively correlated with CDH2, FN1, SNAI1, SNAI2 and TGFBI. The gauge on the right refers to the " $r$ " of Pearson correlation test. Asterisk $(*)$, double asterisk $(* *)$ and triple asterisk $\left({ }^{* * *}\right)$ stand for P-value $<0.05, \mathrm{P}$-value $<0.01$ and $\mathrm{P}$-value $<0.001$, respectively. 
A

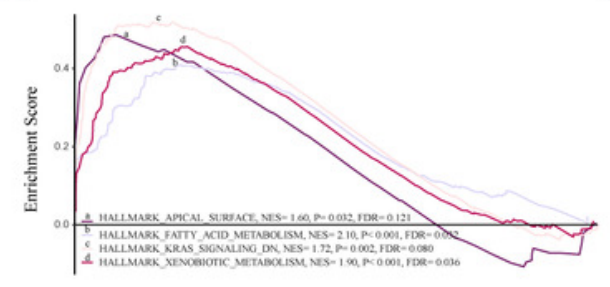

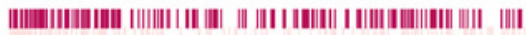

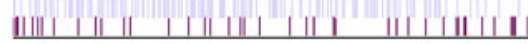

D

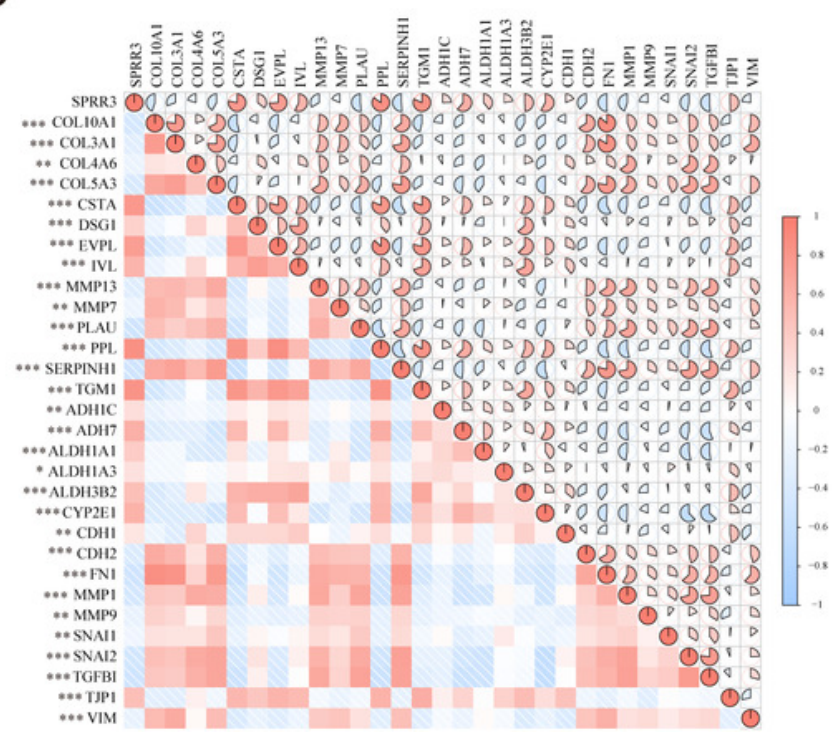

B

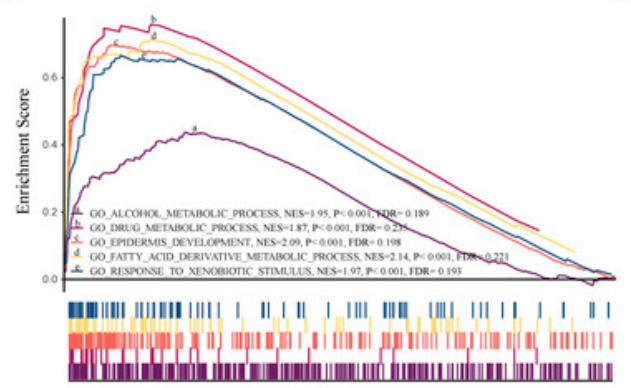

C

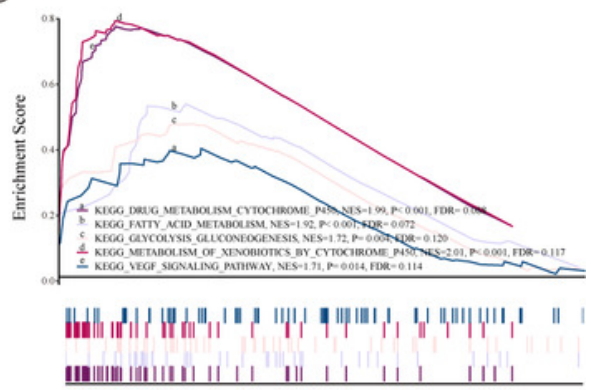

TCGA-OSCC
$\mathbf{E}$

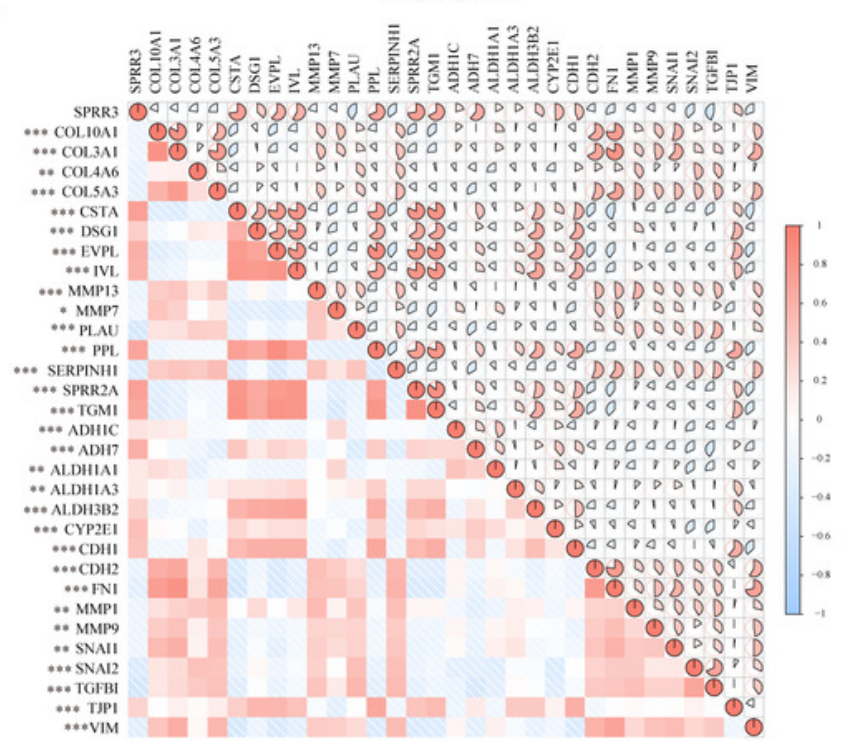




\section{Table $\mathbf{1}$ (on next page)}

The DEGs in the two module clusters screened out by MCODE score 
1 Table 1. The DEGs in the two module clusters screened out by MCODE score

\begin{tabular}{lll}
\hline MCODE Cluster & MCODE Score & Gene ID \\
\hline Cluster 1 & 7 & SPRR2A \\
Cluster 1 & 7 & EVPL \\
Cluster 1 & 7 & PPL \\
Cluster 1 & 7 & DSG1 \\
Cluster 1 & 7 & CSTA \\
Cluster 1 & 7 & TGM1 \\
Cluster 1 & 7 & SPRR3 \\
Cluster 1 & 7 & IVL \\
Cluster 2 & 5 & COL4A6 \\
Cluster 2 & 5 & MMP13 \\
Cluster 2 & 5 & LEPRE1 \\
Cluster 2 & SERPINH1 \\
Cluster 2 & 5 & COL3A1 \\
Cluster 2 & 5 & COL10A1 \\
Cluster 2 & 5 & COL5A3 \\
Cluster 2 & 5 & MMP7 \\
Cluster 2 & 5 & PLAU \\
\hline
\end{tabular}




\section{Table 2 (on next page)}

Cox and LASSO regression analysis for OS in patients with OSCC from GSE42743. 
1 Table 2. Cox and LASSO regression analysis for OS in patients with OSCC from GSE42743.

2 T: primary tumor; N: regional lymph nodes; $(+)$ : selected by LASSO regression; (-): abandoned

3 by LASSO regression

4

\begin{tabular}{|c|c|c|c|c|c|}
\hline \multirow[b]{2}{*}{ Characteristics } & \multicolumn{2}{|c|}{ Univariate analysis } & \multirow[t]{2}{*}{ LASSO } & \multicolumn{2}{|c|}{ Multivariate analysis } \\
\hline & $\begin{array}{c}\text { HR }(95 \% \\
\text { CI) }\end{array}$ & P-value & & $\begin{array}{c}\text { HR }(95 \% \\
\text { CI) }\end{array}$ & P-value \\
\hline $\begin{array}{r}\text { SPRR3 expression } \\
\text { High expression }\end{array}$ & $\begin{array}{c}0.801(0.662- \\
0.969)\end{array}$ & 0.032 & $(+)$ & $\begin{array}{c}0.865 \\
(0.754- \\
0.992)\end{array}$ & 0.037 \\
\hline $\begin{array}{l}\text { CSTA expression } \\
\text { High expression }\end{array}$ & $\begin{array}{c}0.924(0.759- \\
1.126)\end{array}$ & 0.435 & & & \\
\hline $\begin{array}{l}\text { EVPL expression } \\
\text { High expression }\end{array}$ & $\begin{array}{c}0.741(0.480- \\
1.146)\end{array}$ & 0.178 & & & \\
\hline $\begin{array}{l}\text { PPL expression } \\
\text { High expression }\end{array}$ & $\begin{array}{c}0.771(0.616- \\
0.965)\end{array}$ & 0.023 & $(-)$ & & \\
\hline $\begin{array}{l}\text { TGM1 expression } \\
\text { High expression }\end{array}$ & $\begin{array}{c}0.801(0.662- \\
0.969)\end{array}$ & 0.022 & $(-)$ & & \\
\hline $\begin{array}{l}\text { Age (years) } \\
\geq 60 \mathrm{yr}\end{array}$ & $\begin{array}{c}1.001(0.978- \\
1.025)\end{array}$ & 0.920 & & & \\
\hline $\begin{array}{l}\text { Gender } \\
\text { Male }\end{array}$ & $\begin{array}{c}0.694(0.347- \\
1.386)\end{array}$ & 0.300 & & & \\
\hline $\begin{array}{l}\text { Smoking } \\
\text { Current }\end{array}$ & $\begin{array}{c}1.032(0.686- \\
1.552)\end{array}$ & 0.881 & & & \\
\hline $\begin{array}{l}\text { T stage } \\
\\
\mathrm{T}_{3-4}\end{array}$ & $\begin{array}{c}1.39(0.978- \\
1.965)\end{array}$ & 0.067 & & & \\
\hline $\begin{array}{l}\text { N stage } \\
\qquad \text { Non } \mathrm{N}_{0}\end{array}$ & $\begin{array}{c}1.970(1.370- \\
2.832)\end{array}$ & $<0.001$ & $(+)$ & $\begin{array}{c}1.750 \\
(1.202- \\
2.548)\end{array}$ & 0.003 \\
\hline
\end{tabular}




\section{Table 3 (on next page)}

Cox and LASSO regression analysis for OS in patients with OSCC from TCGA. 
1 Table 3. Cox and LASSO regression analysis for OS in patients with OSCC from TCGA.

2 T: primary tumor; N: regional lymph nodes; $(+)$ : selected by LASSO regression

\begin{tabular}{|c|c|c|c|c|c|}
\hline \multirow[b]{2}{*}{ Characteristics } & \multicolumn{2}{|c|}{ Univariate analysis } & \multirow[t]{2}{*}{ LASSO } & \multicolumn{2}{|c|}{ Multivariate analysis } \\
\hline & $\begin{array}{c}\text { HR }(95 \% \\
\text { CI })\end{array}$ & P-value & & $\begin{array}{c}\text { HR }(95 \% \\
\text { CI) }\end{array}$ & P-value \\
\hline $\begin{array}{l}\text { SPRR3 expression } \\
\text { High expression }\end{array}$ & $\begin{array}{c}0.595 \\
(0.371- \\
0.955)\end{array}$ & 0.032 & $(+)$ & $\begin{array}{c}0.544 \\
(0.329- \\
0.899)\end{array}$ & 0.017 \\
\hline $\begin{array}{r}\text { Age (years) } \\
\geq 60 \mathrm{yr}\end{array}$ & $\begin{array}{c}1.086 \\
(0.676- \\
1.745)\end{array}$ & 0.734 & & & \\
\hline $\begin{array}{l}\text { Gender } \\
\text { Male }\end{array}$ & $\begin{array}{c}0.788 \\
(0.448- \\
1.272)\end{array}$ & 0.329 & & & \\
\hline $\begin{array}{c}\text { Alcohol history } \\
\text { Yes }\end{array}$ & $\begin{array}{c}1.363 \\
(0.806- \\
2.305)\end{array}$ & 0.247 & & & \\
\hline $\begin{array}{c}\text { HPV infection } \\
\text { Positive }\end{array}$ & $\begin{array}{c}1.138 \\
(0.518- \\
2.497)\end{array}$ & 0.748 & & & \\
\hline $\begin{array}{c}\text { Radiation therapy } \\
\text { Yes }\end{array}$ & $\begin{array}{c}0.639 \\
(0.398- \\
1.025)\end{array}$ & 0.063 & & & \\
\hline $\begin{array}{l}\text { Histologic grade } \\
\text { Moderate and Poor }\end{array}$ & $\begin{array}{c}1.549 \\
(0.760- \\
3.156)\end{array}$ & 0.228 & & & \\
\hline $\begin{array}{r}\text { Tumor stage } \\
\text { III-IV }\end{array}$ & $\begin{array}{c}1.558 \\
(0.898- \\
2.704)\end{array}$ & 0.115 & & & \\
\hline $\begin{array}{l}\text { T stage } \\
\qquad \mathrm{T}_{3-4}\end{array}$ & $\begin{array}{c}1.607 \\
(0.977- \\
2.643)\end{array}$ & 0.062 & & & \\
\hline $\begin{array}{l}\text { Primary therapy } \\
\text { outcome } \\
\text { Complete } \\
\text { remission }\end{array}$ & $\begin{array}{c}0.282 \\
(0.176- \\
0.453)\end{array}$ & $<0.001$ & $(+)$ & $\begin{array}{c}0.242 \\
(0.147- \\
0.397)\end{array}$ & $<0.001$ \\
\hline $\begin{array}{l}\text { Lymphovascular } \\
\text { invasion } \\
\text { Yes }\end{array}$ & $\begin{array}{c}2.109 \\
(1.266- \\
3.512)\end{array}$ & 0.004 & $(+)$ & $\begin{array}{c}1.690 \\
(0.991- \\
2.881)\end{array}$ & 0.054 \\
\hline $\begin{array}{l}\text { Perineural } \\
\text { invasion } \\
\qquad \text { Yes }\end{array}$ & $\begin{array}{c}1.727 \\
(1.081- \\
2.759)\end{array}$ & 0.022 & $(+)$ & $\begin{array}{c}1.475 \\
(0.857- \\
2.538)\end{array}$ & 0.161 \\
\hline $\begin{array}{l}\text { N stage } \\
\qquad \text { Non } \mathrm{N}_{0}\end{array}$ & $\begin{array}{c}1.833 \\
(1.131- \\
2.971)\end{array}$ & 0.014 & $(+)$ & $\begin{array}{l}1.250 \\
(0.711- \\
2.194)\end{array}$ & 0.438 \\
\hline
\end{tabular}




\section{Table 4 (on next page)}

The relationship between the expression of SPRR3 and multiple clinicopathological factors. 
1 Table 4. The relationship between the expression of SPRR3 and multiple clinicopathological 2 factors.

3 T: primary tumor; $\mathrm{N}$ : regional lymph nodes

\begin{tabular}{|c|c|c|c|c|}
\hline \multicolumn{2}{|l|}{ Characteristics } & SPRR3low & SPRR3high & P-value \\
\hline \multirow[t]{2}{*}{ Gender } & Female & 23 & 28 & 0.390 \\
\hline & Male & 53 & 50 & \\
\hline \multirow[t]{2}{*}{ Age (years) } & $<60 \mathrm{yr}$ & 31 & 35 & 0.515 \\
\hline & $\geq 60 \mathrm{yr}$ & 46 & 42 & \\
\hline \multirow[t]{2}{*}{ Alcohol history } & No & 14 & 59 & 0.011 \\
\hline & Yes & 59 & 47 & \\
\hline \multirow[t]{2}{*}{ HPV infected } & Not infected & 70 & 68 & 0.575 \\
\hline & infected & 6 & 8 & \\
\hline \multirow[t]{2}{*}{ Radiation } & No & 30 & 30 & 1.000 \\
\hline & Yes & 40 & 40 & \\
\hline \multirow[t]{2}{*}{ T stage } & $\mathrm{T}_{1-2}$ & 27 & 35 & 0.403 \\
\hline & $\mathrm{T}_{3-4}$ & 40 & 39 & \\
\hline \multirow[t]{2}{*}{ N stage } & $\mathrm{N}_{0}$ & 18 & 39 & 0.005 \\
\hline & Non $\mathrm{N}_{0}$ & 40 & 31 & \\
\hline \multirow[t]{3}{*}{ Histologic grade } & G1 & 3 & 16 & $<0.001$ \\
\hline & $\mathrm{G} 2$ & 41 & 47 & \\
\hline & G3 & 31 & 14 & \\
\hline \multirow[t]{2}{*}{ Tumor stage } & I-II & 14 & 52 & 0.270 \\
\hline & III-IV & 22 & 53 & \\
\hline \multirow[t]{3}{*}{$\begin{array}{l}\text { Primary therapy } \\
\text { outcome }\end{array}$} & $\begin{array}{l}\text { Progressive } \\
\text { disease }\end{array}$ & 16 & 51 & 0.153 \\
\hline & Complete & & & \\
\hline & remission & 9 & 55 & \\
\hline \multirow[t]{2}{*}{ Perineural invasion } & No & 22 & 42 & 0.016 \\
\hline & yes & 32 & 25 & \\
\hline \multirow[t]{2}{*}{$\begin{array}{l}\text { Lymphovascular } \\
\text { invasion }\end{array}$} & No & 34 & 24 & 0.026 \\
\hline & yes & 43 & 12 & \\
\hline
\end{tabular}

